\title{
The Expression of Key Guidance Genes at a Forebrain Axon Turning Point Is Maintained by Distinct Fgfr Isoforms but a Common Downstream Signal Transduction Mechanism
}

\author{
Jung-Lynn Jonathan Yang, Gabriel E. Bertolesi, Stephanie Dueck, Carrie L. Hehr, and $\mathbb{D}^{\text {Sarah }}$ \\ McFarlane
}

https://doi.org/10.1523/ENEURO.0086-19.2019

Department of Cell Biology and Anatomy, Hotchkiss Brain Institute, Alberta Children's Hospital Research Institute, Cumming School of Medicine, University of Calgary, Calgary, Alberta T2N 4N1, Canada

\begin{abstract}
During development the axons of neurons grow toward and locate their synaptic partners to form functional neural circuits. Axons do so by reading a map of guidance cues expressed by surrounding tissues. Guidance cues are expressed at a precise space and time, but how guidance cue expression is regulated, and in a coordinated manner, is poorly understood. Semaphorins (Semas) and Slits are families of molecular ligands that guide axons. We showed previously that fibroblast growth factor (Fgf) signaling maintains sema3a and slit1 forebrain expression in Xenopus laevis, and these two repellents cooperate to guide retinal ganglion cell (RGC) axons away from the mid-diencephalon and on towards the optic tectum. Here, we investigate whether there are common features of the regulatory pathways that control the expression of these two guidance cues at this single axon guidance decision point. We isolated the sema3a proximal promoter and confirmed its responsiveness to Fgf signaling. Through misexpression of truncated Fgf receptors (Fgfrs), we found that sema3a forebrain expression is dependent on Fgfr2-4 but not Fgfr1. This is in contrast to slit1, whose expression we showed previously depends on Fgfr1 but not Fgfr2-4. Using pharmacological inhibitors and misexpression of constitutively active (CA) and dominant negative (DN) signaling intermediates, we find that while distinct Fgfrs regulate these two guidance genes, intracellular signaling downstream of Fgfrs appears to converge along the phosphoinositol 3-kinase (PI3K)-Akt signaling pathway. A common PI3K-Akt signaling pathway may allow for the coordinated expression of guidance cues that cooperate to direct axons at a guidance choice point.
\end{abstract}

Key words: axon guidance; fibroblast growth factor; MAPK; PI3K/AKT; retinal ganglion cell; signal transduction

\section{Significance Statement}

Little is known about the mechanisms regulating axon guidance cue expression. Fibroblast growth factor (Fgf) signals maintain semaphorin 3a (sema3a) and slit1 expression at a key guidance point for retinal ganglion cell (RGC) axons in the mid-diencephalon. We find that sema3a expression in the forebrain is promoted by a distinct set of Fgfrs, Fgfr2-4, from the Fgfr1 that maintains slit1 levels. Interestingly, despite the differences in Fgfr dependency, the phosphoinositol 3-kinase (PI3K)-Akt signaling pathway is likely a shared downstream regulator of the expression of both guidance cues. These data argue that related but distinct receptors converge on a common signaling mechanism for coordinated control of a map of molecular cues that cooperate at a single guidance choice point to direct axon behavior. 


\section{Introduction}

Axon guidance, cell migration, and cell polarity are key processes required to integrate neurons into functional circuits (O’Donnell et al., 2009; Marín et al., 2010; Seiradake et al., 2016). To form connections, immature neurons respond to molecular cues that determine the position of their cell body and axonal and dendritic processes. These cues include the semaphorins (SEMAs), slits, netrins, and ephrins (Dickson, 2002; Seiradake et al., 2016). While the roles of these molecules are known, the mechanisms that regulate their expression are poorly understood.

The growth cones of axons read redundant molecular cues to navigate through the nervous system and change their direction at axon guidance choice points. For instance, multiple distinct molecules guide the crossing of dorsal commissural interneuron axons at the spinal cord midline, including several members of each of the main axon guidance families (de Ramon Francàs et al., 2017). An interesting question is whether unique or common regulatory pathways control the expression of guidance cues that cooperatively direct axonal trajectories at a single guidance choice point. Our previous work in the Xenopus forebrain provides a model to address this issue. We found that fibroblast growth factor (Fgf) signaling maintains the expression of two guidance cues, sema3a and slit1, which work together to repel retinal ganglion cell (RGC) axons in a caudal direction from an intermediate target within the diencephalon and on toward the optic tectum (Atkinson-Leadbeater et al., 2010); when either Fgf signaling, or both sema3a and slit1 expression, are inhibited, many RGC axons fail to navigate away from this guidance choice point. We identified previously that signaling through Fgf receptor 1 (Fgfr1) maintains forebrain slit1 expression (Yang et al., 2018). By determining through which Fgfr sema3a expression is regulated, and the downstream signaling pathway(s) that control the expression of both guidance cues, we set out to understand how a complete map of guidance cues is established that control the behavior of axons at a select axonal choice point.

Received March 8, 2019; accepted March 11, 2019; First published March 20, 2019

The authors declare no competing financial interests.

Author contributions: J.-L.J.Y., G.E.B., S.D., and S.M. designed research; J.-L.J.Y., G.E.B., S.D., and C.L.H. performed research; J.-L.J.Y., G.E.B., S.D., and S.M. analyzed data; J.-L.J.Y. and S.M. wrote the paper.

This work was supported by the Canadian Institutes of Health Research (CIHR) (S.M.), Alberta Innovates-Health Solutions (S.M.), and the CIHR Training Program in Genetic Determinants of Maternal and Child Health (J.-L.J.Y.).

We thank C. Schuurmans, D. Zinyk, M. Servetnick, A. Nebreda, F. Lang, J. Yang, and A.-F. Burnol for reagents; C. Schuurmans, M. D. Nguyen, and F. Visser for guidance on data analysis; and K. Atkinson-Leadbeater for constructive feedback on this manuscript.

Correspondence should be addressed to Sarah McFarlane at smcfarla@ucalgary.ca.

https://doi.org/10.1523/ENEURO.0086-19.2019

Copyright (C) 2019 Yang et al.

This is an open-access article distributed under the terms of the Creative Commons Attribution 4.0 International license, which permits unrestricted use, distribution and reproduction in any medium provided that the original work is properly attributed.
FGFs regulate cell proliferation, migration, survival, and differentiation (Goetz and Mohammadi, 2013; Ornitz and Itoh, 2015), and through FGFRs activate well-known intracellular signaling cascades to change gene expression, including the mitogen-activated protein kinase (MAPK), phosphoinositol 3-kinase (PI3K)-AKT, and phospholipase C $\gamma($ PLC $\gamma$ ) pathways (Musci et al., 1990; Klint and Claesson-Welsh, 1999; Wiedemann and Trueb, 2000). Here we identify an Fgf-responsive sema3a promoter and show that Fgfr2-4 and not Fgfr1 regulate sema3a expression. Interestingly, while distinct Fgfrs promote sema3a and slit1 (Yang et al., 2018) forebrain expression, they likely employ a common PI3K-Akt signaling pathway. Thus, distinct extrinsic signaling inputs can converge on a common intracellular signal transduction pathway to coordinate the appropriate expression of redundant guidance cues that direct growth cone behavior at a guidance choice point.

\section{Materials and Methods}

\section{Animals}

Xenopus laevis oocytes, collected from adult females (Nasco) injected with human chorionic gonadotrophin (Chorulon, Intervet), were fertilized in vitro. Embryos developed at $16^{\circ} \mathrm{C}$ in $0.1 \times$ Marc's modified Ringer's solution (MMR; $0.1 \mathrm{M} \mathrm{NaCl}, 2 \mathrm{mM} \mathrm{KCl}, 1 \mathrm{mM} \mathrm{MgCl}_{2}$, and $5 \mathrm{mM}$ HEPES, pH 7.5) and were staged according to Faber and Nieuwkoop (1994). Animal protocols were approved by the University of Calgary Animal Care Committee.

\section{Plasmid constructs}

The sema3a upstream flanking nucleotides (-2930 to +63 ; GenBank accession number KP322598) were amplified by PCR from $X$. tropicalis hepatic genomic DNA using the forward primer 5'-GGTGCCTC ATGGGTCAGATTG-3' and reverse primer 5'GTGTGTGCAAAGGCAGCAATG-3'. The first nucleotide transcribed in the sema3a mRNA was assigned the index +1. A PCR-amplified product using Master Mix (Thermo Fisher) was captured into the pCRII-TOPO-TA cloning vector (Life Technologies) according to the manufacturer's instructions. sema3a deletion fragments were isolated by restriction enzymes and ligated upstream of firefly luciferase cDNA in the pGL3 basic (Promega) vector. Primer synthesis and DNA sequencing were conducted at the University of Calgary DNA Core Facility. The Xenopus expression constructs to inhibit Fgfrs were pCS2-DNfgfr 1 (Ueno et al., 1992), pCS108-DNfgfr2, pCS108-sfgfr3, and pCS2MTC-DNfgfr4 (Golub et al., 2000; AtkinsonLeadbeater et al., 2010, 2014). Other constructs used were pCS108-BRAFV600E (courtesy of Carol Schuurmans, University of Toronto; Boehm et al., 2007; Tachibana et al., 2016), pCS2-MEKK1+ and pCS2-MEKK1-KM (provided by Ángel Nebreda, IRB Barcelona; Ben Messaoud et al., 2015), ${ }^{T 308 D / S 473 D} P K B$ and ${ }^{T 308 A / S 473 A} P K B$ (courtesy of Florian Lang, Universität Tübingen; Palmada et al., 2005), AKT-myr (provided by Jing Yang, University of Illinois; Jin et al., 2015), and pEYFP-PLC $\gamma$ and phRlucN1-FGFR1Y766F (provided by Anne-Françoise Burnol, Institut Cochin; Browaeys-Poly et al., 2010). 


\section{Cell culture and luciferase assay}

XTC cells (Pudney et al., 1973; RRID: CVCL_5610), an $X$. laevis fibroblast cell line provided by Manfred Lohka, University of Calgary, were maintained in 60\% Leibovitz's L-15 Medium (Gibco) supplemented with 10\% fetal bovine serum (FBS). Cells were seeded into 96-well plates (Greiner) $24 \mathrm{~h}$ before transfection. Transfections were performed with Lipofectamine 2000 (Life Technologies) in medium without FBS, according with the manufacturer's specifications; each well was co-transfected with 100-ng firefly luciferase (luc) reporter plasmid, 75-ng Renilla plasmid (transfection control, in which the ubiquitous HSVthymidine kinase promoter drives Renilla luciferase expression), and, where required, $50 \mathrm{ng}$ of an expression plasmid or pCS2-GFP (as control). The medium was replaced with medium containing FBS $6 \mathrm{~h}$ after transfection. Where indicated, 3-[3-(2-carboxyethyl)-4-methylpyrrol-2methylidenyl]-2-indolinone (SU5402; Sigma) was added to wells. Cells were harvested for luciferase assay $48 \mathrm{~h}$ after transfection. Luciferase activity was quantified by the Dual-Glo Luciferase Assay System (Promega) as per the manufacturer's instructions on a FilterMax F5 MultiMode Microplate Reader (Molecular Devices). To quantify promoter induction as relative light units, firefly luciferase activity was first normalized against Renilla activity to control for transfection efficiency, and then normalized against the promoterless $p G L 3$ basic vector.

\section{Xenopus brain electroporation}

Brain electroporation of expression plasmids was performed on stage 27/28 embryos anaesthetized in 0.4$\mathrm{mg} / \mathrm{ml}$ tricaine methanesulfonate (Sigma-Aldrich) in $1 \times$ modified Barth's saline (MBS; $0.7 \mathrm{mM} \mathrm{CaCl}_{2}, 5 \mathrm{mM}$ HEPES, $1 \mathrm{mM} \mathrm{KCl,} 1 \mathrm{mM} \mathrm{MgSO}_{4}$, $88 \mathrm{mM} \mathrm{NaCl}$, and 2.5 $\mathrm{mM} \mathrm{NaHCO}_{3}$; $\mathrm{pH}$ 7.8; Haas et al., 2002; Chen et al., 2007). Briefly, a $1-\mu \mathrm{g} / \mu \mathrm{l}$ plasmid DNA solution was microinjected into the central forebrain ventricle using a Picospritzer II (General Valve). Platinum electrodes, spaced $3 \mathrm{~mm}$ apart and connected to a Grass Technologies S44 system, were placed on either side of the embryo's head to deliver 10 50-ms 35-V pulses. Electroporated embryos were reared in $0.1 \times$ MMR for development to stage 32 .

\section{In situ hybridization (ISH)}

Digoxigenin (DIG)-labeled and fluorescein-labeled riboprobes were prepared and used for ISH as described previously (Atkinson-Leadbeater et al., 2010; Yang et al., 2018). Briefly, riboprobes were transcribed in vitro using SP6 or T7 polymerase (Roche), DIG-labeled or fluorescein-labeled ribonucleotides (Roche), and linearized plasmid templates pBSK-xfgfr1, pBSK-xBek-ec, pBSK-xfgfr3, pBSK-xfgfr4, pCRII-xsema3A, and pCMV-SPORT6-slit1 (Golub et al., 2000; Atkinson-Leadbeater et al., 2009, 2010, 2014). The specificity of all riboprobes was assessed through sense controls. For color development of wholemount ISH, tissues were incubated with anti-DIG alkaline phosphataseconjugated Fab fragments (Roche catalog \#11 093274 910; RRID: AB_2313640) and stained with BM Purple (Roche; Sive et al., 2000). For double fluorescent ISH (dFISH) on sectioned tissue, samples were incubated with anti-DIG peroxidase-conjugated (Roche catalog \#11 207733 910;
RRID: AB_514500) or anti-fluorescein peroxidase-conjugated (Roche catalog \#11 426346 910; RRID AB_840257) Fab fragments and stained with the TSA Plus Fluorescein Evaluation kit (PerkinElmer) and the TSA Cyanine 3 System (PerkinElmer).

\section{Immunohistochemistry}

Embryos were fixed in 4\% paraformaldehyde, cryoprotected in $30 \%$ sucrose solution, and mounted in OCT compound (Tissue Tek, Sakura Finetek, Inc.) for cryosectioning to $12-\mu \mathrm{m}$ thickness. The tissues were permeabilized in 0.5\% Triton X-100 (Sigma-Aldrich) and blocked in $5 \%$ goat serum (Thermo Fisher) in PBS for 30 min at room temperature. Primary antibodies (1:500 dilution) were against hemagglutinin (HA; Covance catalog \#MMS101P; RRID: AB_2565006), green fluorescent protein (GFP; Invitrogen catalog \#A11120; RRID: AB_221568), and myc (Santa Cruz Biotechnology catalog \#sc-789; RRID: AB_631274). The secondary antibodies conjugated to Alexa Fluor 488 (1:1000 dilution) were goat anti-mouse (Abcam catalog \#ab150113; RRID: AB_2576208) and goat anti-rabbit (Abcam catalog \#ab150077; RRID: AB_2630356). Nuclei were stained with 4,6-diamidino-2phenylindole (DAPI; 1:1000; Life Technologies).

\section{In vivo-exposed brain preparation}

The skin and dura overlying the left side of the brain of stage 32 or stage 33/34 embryos were removed to expose the entire anterior brain to as far as the posterior optic tectum (Chien et al., 1993). Embryos were incubated in specific inhibitors against Fgfrs, Mek, PI3K, and PLC $\gamma$ in $1 \times \mathrm{MBS}$ at room temperature for $5 \mathrm{~h}$ : SU5402, 1,4diamino-2,3-dicyano-1,4-bis(o-aminophenylmercapto) butadiene monoethanolate (U0126; Calbiochem), 2-(4morpholinyl)-8-phenyl-4H-1-benzopyran-4-one (LY294002; Calbiochem), and 1-[6-[((17 $\beta)-3-$ methoxyestra-1,3,5[10]-trien-17-yl)amino]hexyl]-1Hpyrrole-2,5-dione (U73122; Calbiochem), respectively. The control incubation was $1 \times$ MBS supplemented with $0.2 \%$ dimethyl sulfoxide (DMSO). For ISH, embryos were fixed in 4\% paraformaldehyde. For RT-qPCR, the anterior brains were collected into TRIzol (Life Technologies). For optic tract tracing, the RGC axons were anterogradely labeled with horseradish peroxidase (HRP) at stage 40 (Atkinson-Leadbeater et al., 2010). To quantify the axon stall phenotype, the width of the optic tract post middiencephalic turn was represented as a ratio to the width of the optic tract at the mid-diencephalic turn.

\section{Reverse transcription (RT)-PCR}

Total RNA was extracted from $X$. laevis forebrains and XTC cells using TRIzol and chloroform and purified with the GeneJET RNA Purification kit (Thermo Fisher). RNA quality was assessed on the Agilent 2200 TapeStation (University of Calgary Center for Health Genomics and Informatics). The cDNA template was prepared from 100-ng total RNA in 20- $\mu$ l reactions using SuperScript II Reverse Transcriptase (Life Technologies) and primed with oligodT (Life Technologies). The composition of quantitative RT-PCR (RT-qPCR) reactions was 3- $\mu$ l cDNA 
Table 1. RT-qPCR primers and their properties for quantifying gene expression

\begin{tabular}{|c|c|c|c|c|}
\hline Gene & Primers & Amplicon & Efficiency & $\begin{array}{l}\text { Amplicon } \\
T_{\text {melt }}\left({ }^{\circ} \mathrm{C}\right)\end{array}$ \\
\hline actb* & $\begin{array}{l}\text { Forward, } \\
\text { GTTGATAATGGATCTGGTATGTGC } \\
\text { Reverse, } \\
\text { ATTCCAACCATGACACCCTGA }\end{array}$ & $\begin{array}{l}\text { Nucleotides 89-201, GenBank accession } \\
\text { number NM_001088953.1 }\end{array}$ & 0.9401 & 83.0 \\
\hline dynll1 $\diamond$ & $\begin{array}{l}\text { Forward, } \\
\text { TGCTACTCAGGCACTGGAGA } \\
\text { Reverse, } \\
\text { AATTCCTTCCCACAATGCAA }\end{array}$ & $\begin{array}{l}\text { Nucleotides 127-239, GenBank accession } \\
\text { number NM_001171695.1 }\end{array}$ & 0.9576 & 77.5 \\
\hline sema3a & $\begin{array}{l}\text { Forward, } \\
\text { TGAAGAACGGGGAAGACTTTATG } \\
\text { Reverse, } \\
\text { TGTTACAGGCCACAATATCTTTTG }\end{array}$ & $\begin{array}{l}\text { Nucleotides 189-288, GenBank accession } \\
\text { number NM_001085855.1 }\end{array}$ & 0.9738 & 78.5 \\
\hline slit1* & $\begin{array}{l}\text { Forward, } \\
\text { TGCTGAGCGTAAACTTTGTGG } \\
\text { Reverse, TCCGCTGTTGACACCTGAAG }\end{array}$ & $\begin{array}{l}\text { Nucleotides 3756-3855, GenBank accession } \\
\text { number NM_001087109.1 }\end{array}$ & 0.9931 & 81.0 \\
\hline slit2 & $\begin{array}{l}\text { Forward, } \\
\text { CCAGGAGCATTCTCACCATACA } \\
\text { Reverse, } \\
\text { TAGAGAACAAGGGAGTTGAGTGA }\end{array}$ & $\begin{array}{l}\text { Nucleotides 1510-1628, GenBank accession } \\
\text { number NM_001087668.1 }\end{array}$ & 0.9220 & 80.0 \\
\hline \multirow[t]{2}{*}{$\begin{array}{l}\text { spry1 } \\
\mathrm{a} / \mathrm{b} *\end{array}$} & Forward, CAACATGGCATTGGTGGTTCAT & $\begin{array}{l}\text { Nucleotides 16-110, GenBank accession } \\
\text { number NM_001137585.1; nucleotides 72-166, } \\
\text { GenBank accession number NM_001159681.1 }\end{array}$ & 0.9490 & 82.0 \\
\hline & $\begin{array}{l}\text { Reverse, } \\
\text { TTTGATCTGATCCAAGGACAAGATAG }\end{array}$ & & & \\
\hline tubb* & $\begin{array}{l}\text { Forward, } \\
\text { GACCCTITGGACAGATTTTCAGG }\end{array}$ & $\begin{array}{l}\text { Nucleotides 300-405, GenBank accession } \\
\text { number NM_001087257.1 }\end{array}$ & 0.9635 & 81.5 \\
\hline
\end{tabular}

*Yang et al. (2018)

$\diamond$ Brunsdon (2015)

solution, $1 \times$ QuantiTect SYBR Green PCR kit (QIAGEN), $417 \mathrm{nM}$ each of forward and reverse primers, and water up to $20 \mu \mathrm{l}$. RT-PCR primers for fgfr 1-4 and RT-qPCR primers (Table 1) for slit1 and spry1, and the reference genes $\beta$-actin (actb), tubulin $\beta$ class I (tubb), and light chain dynein (dyn/l1), were validated previously (Brunsdon, 2015; Yang et al., 2018). We also validated RT-qPCR primers for sema3a and slit2 for high efficiency and specificity (data not shown). RT-qPCR reactions were run on the CFX Connect Real-Time PCR Detection System (BioRad) and the CFX Manager Software v3.1 (Bio-Rad). Each RT-PCR and RT-qPCR reaction was thermocycled as initial denature $\left(95^{\circ} \mathrm{C}, 10 \mathrm{~min}\right)$, denature $\left(95^{\circ} \mathrm{C}, 15 \mathrm{~s}\right)$, anneal $\left(54^{\circ} \mathrm{C}, 35 \mathrm{~s}\right)$, and elongation $\left(72^{\circ} \mathrm{C}, 30 \mathrm{~s}\right)$ for a total of 40 cycles. The RT-qPCR product was heated to $95^{\circ} \mathrm{C}$ in $0.5^{\circ} \mathrm{C}$ increments for melt curve analysis.

\section{Western blotting}

The brains of pharmacologically treated embryos and XTC cells were collected in $100 \mu \mathrm{L}$ homogenization buffer [10\% glycerol, $137 \mathrm{mM} \mathrm{NaCl}, 1.5 \mathrm{mM} \mathrm{Na}_{3} \mathrm{VO}_{4}, 1 \% \mathrm{NP} 40$, $0.1 \%$ sodium dodecyl sulfate (SDS), $20 \mathrm{mM}$ Tris; $\mathrm{pH} 8.0$, and Roche protease inhibitor mix diluted 1:100]; $10 \mu \mathrm{l}$ of the homogenate was added to $200 \mu \mathrm{l}$ of protein assay dye (Bio-Rad) diluted 1:4 and measured by the FilterMax F5 Multi-Mode Microplate Reader. Sample buffer (2\% mercaptoethanol, $20 \%$ glycerol, and $4 \%$ SDS) was added to $5-\mu \mathrm{g}$ protein samples loaded onto a $10 \%$ polyacrylamide gel. Samples were transferred to a PVDF membrane (Bio-Rad) and incubated in blocking solution containing primary antibodies (1:1000 dilution); rabbit anti-pERK (Cell Signaling
Technology catalog \#4370; RRID: AB_2315112), anti-pAKT (Cell Signaling Technology catalog \#4060; RRID: AB_2315049), anti-GAPDH (Cell Signaling Technology catalog \#5174; RRID: AB_10622025), anti-SEMA3A (Abcam catalog \#ab23393; RRID: AB_447408), and goat anti-actin antibodies (Abcam catalog \#ab8229; RRID: AB_306374) at room temperature for $1 \mathrm{~h}$. ECL solution (GE Healthcare Bioscience) was used according to the manufacturer's instructions after incubation with the secondary antibodies (1:500 dilution) HRP-conjugated goat anti-rabbit (Jackson ImmunoResearch catalog \#111-035-144; RRID: AB_2307391) or donkey anti-goat (Jackson ImmunoResearch catalog \#705-035-147; RRID: AB_2313587).

\section{Image processing and analysis}

Microscope images were captured on the AxioCam HRc software (Carl Zeiss) on the Stemi SV II stereomicroscope (Carl Zeiss) and processed minimally in Photoshop (Adobe, 2017.0.0 release) for brightness and contrast. To evaluate co-expression of fgfr and slit1 mRNAs with sema3a mRNA, ImageJ (1.51r; Schneider et al., 2012) was used to analyze the extent of overlap in the area of the gene expression domains in images of dFISH brain sections. The area of the fgfr or slit1 domain is reported as a fraction of the area of the sema3a domain.

\section{Statistical analysis}

For all quantitative datasets, the bars represent the mean with error bars representing the SEM from a total of $n$ replicates in $N$ independent experiments. After considering normality, datasets involving a control group and a 
Table 2. Statistical table

\begin{tabular}{|c|c|c|c|}
\hline & Data structure & Type of test & $\begin{array}{l}95 \% \text { confidence } \\
\text { interval }\end{array}$ \\
\hline a & Normal distribution & $\begin{array}{l}\text { One-way ANOVA, Bonferroni's } \\
\text { multiple comparison post hoc test }\end{array}$ & {$[-20.96,-1.920]$} \\
\hline b & Normal distribution & Student's $t$, two-tailed & {$[-1.786,66.14]$} \\
\hline c & Normal distribution & Student's $t$, two-tailed & {$[-69.34,-7.449]$} \\
\hline$d$ & Normal distribution & $\begin{array}{l}\text { One-way ANOVA, Dunnett's multiple } \\
\text { comparison post hoc test }\end{array}$ & {$[-23.38,32.66]$} \\
\hline e & Normal distribution & $\begin{array}{l}\text { One-way ANOVA, Dunnett's multiple } \\
\text { comparison post hoc test }\end{array}$ & {$[18.43,80.26]$} \\
\hline f & Normal distribution & $\begin{array}{l}\text { One-way ANOVA, Dunnett's multiple } \\
\text { comparison post hoc test }\end{array}$ & {$[18.77,77.36]$} \\
\hline g & Normal distribution (central limit theorem) & Randomization test, 2000 iterations & {$[0.155,3.345]$} \\
\hline $\mathrm{h}$ & Normal distribution (central limit theorem) & Randomization test, 2000 iterations & {$[0.048,1.645]$} \\
\hline i & Normal distribution (central limit theorem) & Randomization test, 2000 iterations & {$[0.077,1.438]$} \\
\hline j & Normal distribution (central limit theorem) & Randomization test, 2000 iterations & {$[0.062,1.240]$} \\
\hline $\mathrm{k}$ & Normal distribution (central limit theorem) & Randomization test, 2000 iterations & {$[0.049,13.756]$} \\
\hline I & Normal distribution (central limit theorem) & Randomization test, 2000 iterations & {$[0.056,9.819]$} \\
\hline $\mathrm{m}$ & Normal distribution (central limit theorem) & Randomization test, 2000 iterations & {$[0.022,1.931]$} \\
\hline $\mathrm{n}$ & Normal distribution (central limit theorem) & Randomization test, 2000 iterations & {$[0.019,1.771]$} \\
\hline o & Normal distribution (central limit theorem) & Randomization test, 2000 iterations & {$[0.033,1.649]$} \\
\hline $\mathrm{p}$ & Normal distribution (central limit theorem) & Randomization test, 2000 iterations & {$[0.367,1.650]$} \\
\hline q & Normal distribution (central limit theorem) & Randomization test, 2000 iterations & {$[0.011,0.922]$} \\
\hline r & Normal distribution (central limit theorem) & Randomization test, 2000 iterations & {$[0.024,1.198]$} \\
\hline s & Normal distribution (central limit theorem) & Randomization test, 2000 iterations & {$[0.361,1.499]$} \\
\hline $\mathrm{t}$ & Normal distribution (central limit theorem) & Randomization test, 2000 iterations & {$[0.016,22.586]$} \\
\hline u & Normal distribution (central limit theorem) & Randomization test, 2000 iterations & {$[0.022,1.931]$} \\
\hline $\mathrm{v}$ & Normal distribution (central limit theorem) & Randomization test, 2000 iterations & {$[0.024,1.564]$} \\
\hline w & Normal distribution (central limit theorem) & Randomization test, 2000 iterations & {$[0.662,1.628]$} \\
\hline$x$ & Normal distribution (central limit theorem) & Randomization test, 2000 iterations & {$[0.473,2.347]$} \\
\hline y & Normal distribution (central limit theorem) & Randomization test, 2000 iterations & {$[0.676,9.860]$} \\
\hline z & Normal distribution (central limit theorem) & Randomization test, 2000 iterations & {$[0.595,2.040]$} \\
\hline aa & Normal distribution (central limit theorem) & Randomization test, 2000 iterations & {$[0.570,2.160]$} \\
\hline$a b$ & Normal distribution (central limit theorem) & Randomization test, 2000 iterations & {$[0.461,1.626]$} \\
\hline ac & Normal distribution (central limit theorem) & Randomization test, 2000 iterations & {$[0.360,1.574]$} \\
\hline ad & Normal distribution (central limit theorem) & Randomization test, 2000 iterations & {$[0.432,1.370]$} \\
\hline ae & Normal distribution (central limit theorem) & Randomization test, 2000 iterations & {$[0.315,1.722]$} \\
\hline af & Normal distribution (central limit theorem) & Randomization test, 2000 iterations & {$[0.450,1.469]$} \\
\hline ag & Normal distribution (central limit theorem) & Randomization test, 2000 iterations & {$[0.281,1.182]$} \\
\hline ah & Normal distribution (central limit theorem) & Randomization test, 2000 iterations & {$[0.283,1.425]$} \\
\hline ai & Normal distribution (central limit theorem) & Randomization test, 2000 iterations & {$[0.469,1.389]$} \\
\hline aj & Normal distribution (central limit theorem) & Randomization test, 2000 iterations & {$[0.652,3.255]$} \\
\hline ak & Normal distribution (central limit theorem) & Randomization test, 2000 iterations & {$[0.480,2.787]$} \\
\hline al & Normal distribution (central limit theorem) & Randomization test, 2000 iterations & {$[0.856,4.190]$} \\
\hline $\mathrm{am}$ & Normal distribution (central limit theorem) & Randomization test, 2000 iterations & {$[0.812,1.928]$} \\
\hline an & Normal distribution (central limit theorem) & Randomization test, 2000 iterations & {$[0.530,30.724]$} \\
\hline ao & Normal distribution (central limit theorem) & Randomization test, 2000 iterations & {$[0.294,1.836]$} \\
\hline ap & Normal distribution & Student's $t$, two-tailed & {$[0.480,0.694]$} \\
\hline aq & Normal distribution & Student's $t$, two-tailed & {$[0.346,0.617]$} \\
\hline
\end{tabular}

treatment group were analyzed on GraphPad Prism 7 (RRID: SRC_002798) by using unpaired, two-tailed Student's $t$ test. Datasets involving a control group and multiple treatment groups were analyzed on GraphPad Prism 7 using one-way ANOVA with Dunnett's or Bonferroni's post hoc test. RT-qPCR data were analyzed on the Relative Expression Software Tool (REST) 2009 V2.0.13 (QIAGEN). The expression of spry1, sema3a, and slit1/2 were normalized against the stably expressed reference genes $a c t b, t u b b, d y n l l 1$. For all statistical analyses, the threshold of significance was set at 0.05 . The details of the statistical analyses are shown in Table 2.

\section{Results}

\section{fgfr1-4 and sema3a co-express in the embryonic forebrain}

Sema3a and Slit1 cooperate as repellents to guide RGC axons past the mid-diencephalon and toward the midbrain target, the optic tectum. We found previously that Fgf signaling in the Xenopus forebrain maintains the expression of both guidance genes (Atkinson-Leadbeater et al., 2010), with Fgfr1 critical for regulating slit1 expression (Yang et al., 2018). To investigate whether Fgfr1 also regulates forebrain sema3a, we first asked which fgfr 
isoforms are present alongside sema3a domains. We performed dFISH on transverse sections through the $X$. laevis forebrain (Fig. 1A) to observe fgfr-sema3a co-expression at stage 32, when RGC axons are growing toward the sema3a and slit 1 forebrain domains (Atkinson-Leadbeater et al., 2010). Of note, due to the distinct spatial expression patterns of fgfrs in the forebrain, we show representative sections where maximal co-expression occurred along the rostrocaudal axis of sema3a with the particular fgfr isoform. Unlike slit1, whose expression in the forebrain overlapped significantly only with that of fgfr 1 (Yang et al., 2018), the dFISH signal for all four fgfr isoforms coincided with some portion of the sema3a expression domain. For instance, both fgfr 1 and sema3a were expressed by the cells surrounding the ventricle of the forebrain, and fgfr2 was expressed alongside sema3a at the apical (ventricular) face of the roof plate. In analysis of forebrain sections, the fgfr $1 / 2$ signals covered $85 \%$ and $2 \%$, respectively, of the sema3a domain. sema3a expression overlapped with both fgfr $3 / 4$ in cells surrounding the ventricle. Overlap was not complete, in that the fgfr 3 region extended more ventrally to coincide with $57 \%$ of the sema3a domain, and that of fgfr 4 covered $90 \%$ of the sema3a domain within the dorsal neural tube. Altogether, the co-expression of sema3a with fgfrs supports a model whereby Fgfr signaling regulates cell-autonomously sema3a expression in the forebrain.

Since Sema3a and Slit1 cooperate to guide RGC axons (Atkinson-Leadbeater et al., 2010), we compared the spatial aspects of sema3a and slit1 expression. By dFISH, we visualized the expression patterns of both genes in transverse sections along the rostrocaudal axis of the brain. Interestingly, given their cooperative role in RGC axon guidance, the sema3a and slit1 domains were generally non-overlapping; sema3a expression was localized toward the ventricular face of the neural tube, while slit1 mRNA was at the pial surface and in the floor plate (Fig. $1 B)$. Of note, there was slight sema3a-slit1 co-expression in cells at the interface of the expression domains of the two guidance genes, seen in the telencephalon, midbrain, and hindbrain coinciding with $17 \%, 8 \%$, and $17 \%$, respectively, of the sema3a domain. The distinct fgfrsema3a and fgfr-slit1 (Yang et al., 2018) co-localization patterns together with the non-overlapping sema3a-slit1 expression in the forebrain suggest that unique regulatory mechanisms exist to control these two guidance cues.

\section{Fgf signaling induces sema3a promoter activity}

To examine the mechanisms of sema3a transcriptional regulation, we isolated the sema3a 5'-flanking sequence, and performed a deletion analysis to characterize the proximal promoter elements. Deletion fragments from the 5'- and 3'-end of the $-2930+63$ sema3a sequence were inserted upstream of the luciferase (Iuc) reporter gene (Fig. 2A). These deletion constructs were co-transfected alongside Renilla, to normalize transfection efficiency, into XTC cells, an X. laevis fibroblast cell line. The XTC line was found to express Sema3a by Western blotting (Fig. 2B), suggesting sema3a regulatory mechanisms are in place. In the identified sema3a promoter there appeared to be silencing activity within -1102 to -784 , as exclusion of these nucleotides increased reporter induction $(n=9$ and $N=3$ for all bars, compare constructs $-1102+63$ and $-784+63, p<0.05$; Fig. $2 C$; row a, Table 2). The shortest deletion fragment with promoter activity was $-677-150$. Since the $-677-450$ fragment did not possess promoter activity, the sema3a core promoter was therefore located within nucleotides -450 to -150 .

Next, we examined which fgfr isoforms could regulate the activity of the sema3a promoter. To confirm that the upstream regulatory sequence contained the necessary elements for responsiveness to Fgf signaling, we explored whether Fgfr signaling acted on the identified $-2930+63$ sema3a sequence in XTC cells. Of note, by RT-PCR we found that XTC cells express fgfr1-3, but not fgfr4 (Fig. $2 D$ ). Pharmacological inhibition of Fgfrs with $100 \mu \mathrm{M}$ of Fgfr inhibitor SU5402 did not affect the activity of a heparanase promoter construct (Bertolesi et al., 2011; $n=12$ and $N=2$ for both bars, $p=0.062$; Fig. $2 E$; row $b$, Table 2), but repressed significantly the activity of the $-2930+63$ sema3a::/uc reporter $(n=12$ and $N=3$ for both bars, $p=0.018$; Fig. $2 F$; row $c$, Table 2). To identify the contribution of each Fgfr to sema3a transactivation, expression vectors for truncated Fgfrs (Ueno et al., 1992; Golub et al., 2000; Atkinson-Leadbeater et al., 2010, 2014) were co-transfected into XTC cells along with $-2930+63$ sema3a::/uc and Renilla. The dominant negative (DN) Fgfrs form non-functional heterodimers with wild-type Fgfrs, thereby eliminating signaling from their wild-type counterparts (Ueno et al., 1992), while soluble Fgfr3 (sFgfr3) sequesters specific Fgfs from binding endogenous Fgfrs (Fukuchi-Shimogori and Grove, 2001). Blocking Fgfr1 did not affect significantly the sema3a promoter activity (DN fgfr $1 n=16$ wells and $N=7$ vs control $n=30$ wells and $N=7, p>0.05$; Fig. 2G; row $d$, Table 2), while DN fgfr2 and sfgfr3 lowered sema3a promoter activity to $50.7 \%(n=12$ wells and $N=7, p<0.01$; Fig. 2G; row e, Table 2) and $51.9 \%(n=14$ wells and $N=$ 7, $p<0.01$; Fig. 2G; row f, Table 2) of basal activity, respectively. Therefore, in XTC cells, the sema3a promoter is regulated in an Fgfr isoform-dependent fashion.

To determine whether similar Fgfr2/3-sema3a regulatory relationship occurs in the embryonic brain, expression vectors for DN fgfr1/2/4 and sfgfr3 were electroporated into stage $27 / 28$ X. laevis forebrains (Fig. $3 A$ ). Twenty-four hours after electroporation, the forebrains were collected for ISH and RT-qPCR to spatially and quantitatively assess gene expression, respectively. The DN fgfr1/2 constructs had strong expression in the electroporated half of the forebrain, as assayed by FISH (Fig. 3B,C), as did sfgfr 3 and DN fgfr 4 vectors (data not shown). We first assessed sema3a expression in electroporated brains by ISH (Fig. 3D-F). As in XTC cells, expression of DN fgfr1 ( $n=20$ brains, $N=3$ ) did not noticeably affect sema3a compared to the pCS2-GFP control ( $n=37$ brains, $N=3$ ), but was shown previously to repress forebrain slit1 expression (Yang et al., 2018). In contrast, by ISH, sema3a expression in DN fgfr2 electroporated brains was decreased relative to control $(n=$ $16 / 24$ and $N=3$ DN fgfr2 brains). RT-qPCR verified that, 
A
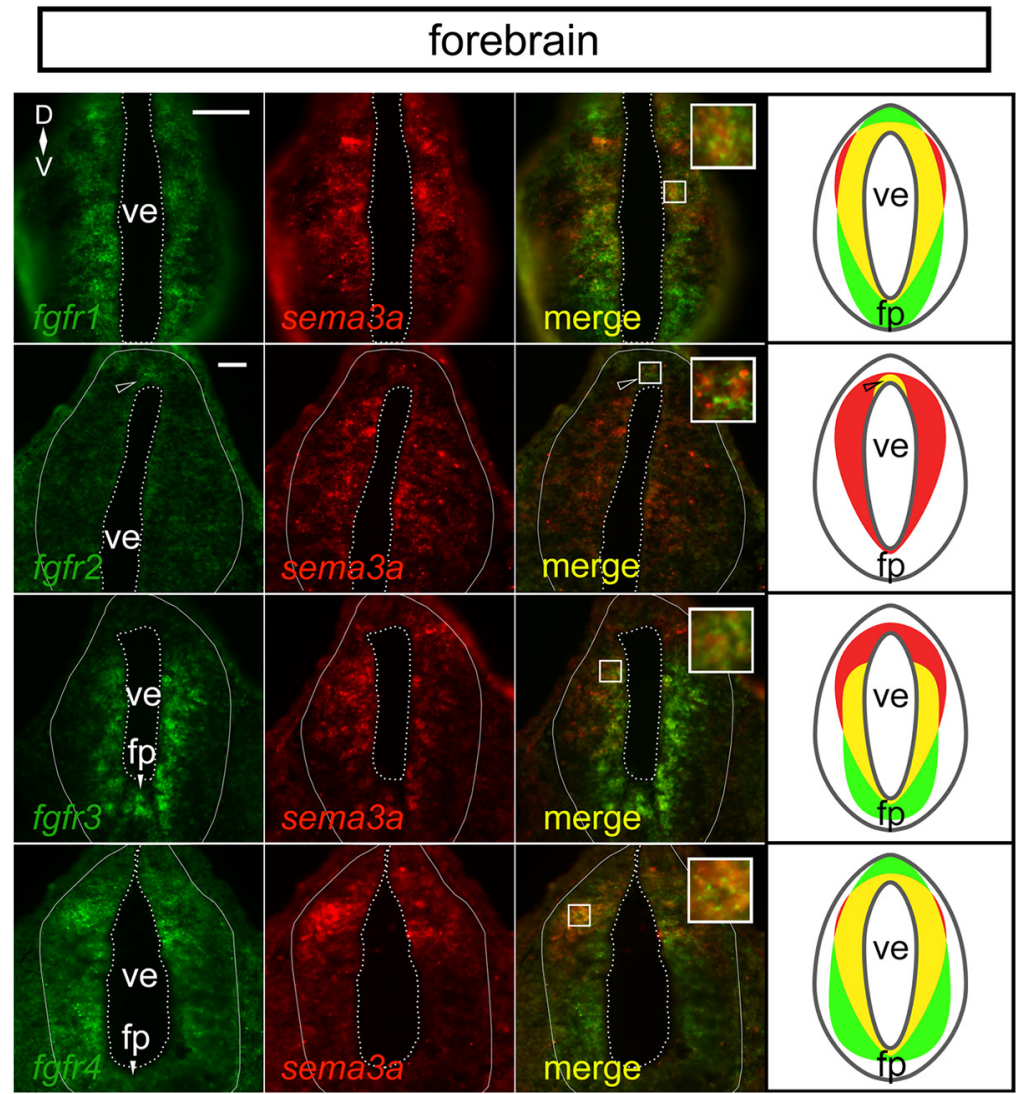

B
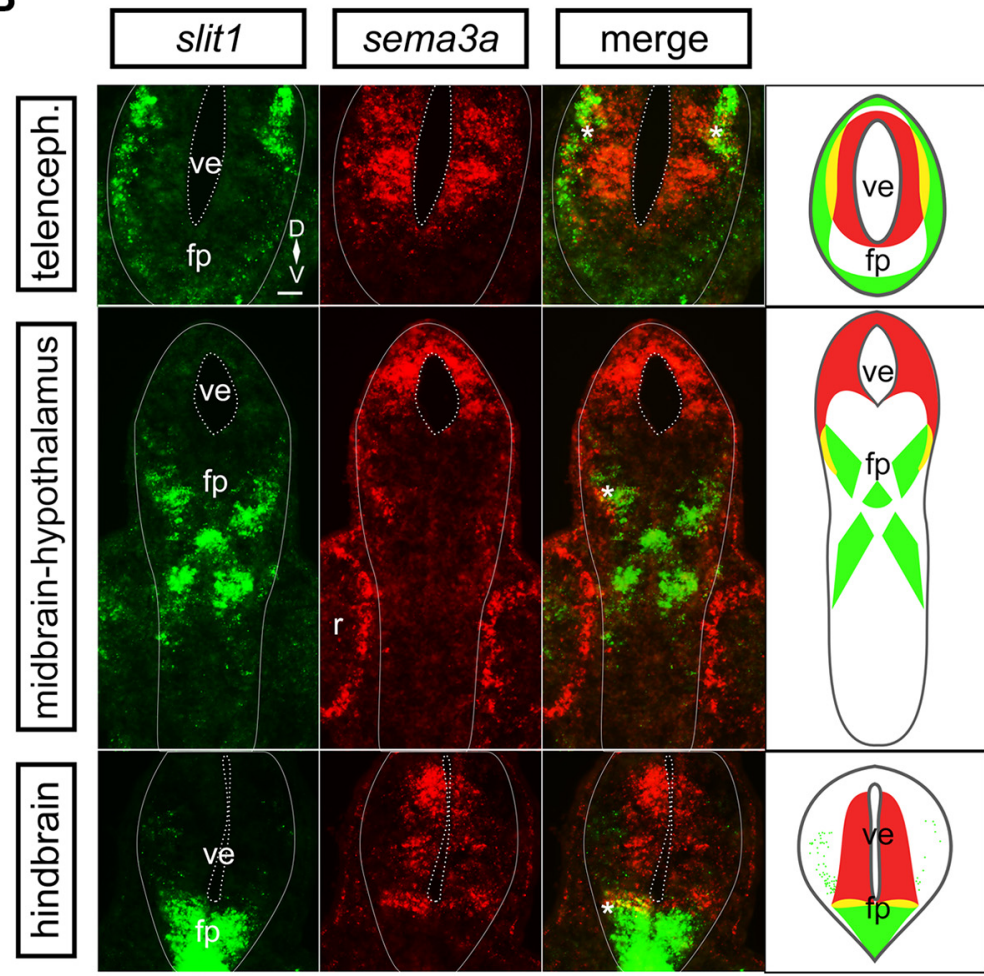

Figure 1. sema3a is co-expressed with fgfrs in the embryonic forebrain, but in complementary domains to slit1. $\boldsymbol{A}$, dFISH on transverse sections through the forebrain using specific antisense riboprobes against fgfr 1-4 (green) and sema3a (red). There is co-expression (yellow) of sema3a with all fgfrs in the ventricular zone of the forebrain. The insets reveal co-localization of dFISH signal. 


\section{continued}

The rightmost column shows cartoons of the fgfr (green) and sema3a (red) domains, with co-expression in yellow. The fgfr2 region of co-expression is restricted to the dorsal ventricular zone (unfilled arrowhead). $\boldsymbol{B}, \mathrm{dFISH}$ on transverse sections through the forebrain by using specific antisense riboprobes against slit1 (green) and sema3a (red). sema3a is expressed by cells around the forebrain ventricle, whereas slit1 is localized to the pial cells and the floor plate of the neural tube. There is some limited co-expression (yellow) of sema3a and slit1 at the interface (asterisk) of the two expression domains. The rightmost column shows cartoons of the slit1 (green) and sema3a (red) domains, with co-expression in yellow. Scale bars, $50 \mu \mathrm{m}$. fp, floor plate; ve, ventricle.

compared to control electroporation $(n=121$ brains, $N=$ 4; Fig. 3G), DN fgfr1 did not affect sema3a significantly ( $n$ $=24$ brains, $N=4, p=0.754$; row $\mathrm{g}$, Table 2), whereas DN fgfr2, sfgfr3, and DN fgfr4 decreased sema3a induction to $40.6 \%(n=20$ brains, $N=3, p=0.005$; row h, Table 2), $47.1 \%(n=30$ brains, $N=4, p=0.004$; row i, Table 2), and $37.9 \%(n=31$ brains, $N=4, p<0.001$; row $\mathrm{j}$, Table 2), respectively. Of note, all the truncated Fgfrs were effective at blocking Fgf signaling as seen by the decreased expression of spry 1 by RT-qPCR, a feedback regulator of Fgfr signaling (Hacohen et al., 1998; AtkinsonLeadbeater et al., 2009). In agreement with the XTC cell line data, we find that Fgfr2/3 regulate forebrain sema3a. While Fgfr 4 regulates sema3a in the forebrain, fgfr 4 mRNA is absent in XTC cells. In contrast, Fgfr1 appears to be the sole Fgfr regulating slit1 in the forebrain (Yang et al., 2018). Thus, sema3a and slit1 mRNA levels are maintained by distinct Fgfrs in the embryonic Xenopus forebrain.

\section{Pharmacological blockade of PI3K signaling inhibits both sema3a and slit1 expression}

Because we find that Fgf signaling regulates sema3a and slit1 transcription via different Fgfr isoforms, we next asked whether distinct downstream signaling cascades are involved. The candidate pathways downstream of FGFRs are the MAPK, PI3K-AKT, and PLC $\gamma$ cascades (Alessi et al., 1996; Alessi and Cohen, 1998; Klint and Claesson-Welsh, 1999; Rubinfeld and Seger, 2004; Goetz and Mohammadi, 2013; Ornitz and Itoh, 2015; Nakamura and Fukami, 2017; Fig. 4A). To determine which of these signaling pathways are relevant for sema3a and slit1 expression, pharmacological inhibitors against each pathway were applied to exposed brain preparations of stage 32 X. laevis embryos (Chien et al., 1993; AtkinsonLeadbeater et al., 2010). The pharmacological approach has the advantage that pathways can be inhibited for a short time period (hours), where effects on gene transcription are likely directly attributable to changes in the activity of the pathway. The skin and dura overlying the brain on the left side of the embryo were surgically removed and the embryo incubated for $5 \mathrm{~h}$ in a control solution or media containing the appropriate inhibitor. Notably, the Fgfr inhibitor SU5402 decreases sema3a and slit1 expression over this same time period (Atkinson-Leadbeater et al., 2010). We used the Mek inhibitor U0126 (100 $\mu \mathrm{M})$ to inhibit the Erk MAPK pathway, LY294002 (25 $\mu$ M) to block PI3K, and U73122 (10 $\mu \mathrm{M})$ to inhibit PLC $\gamma$ (Fig. 4A). These concentrations were shown previously to be effective in Xenopus and zebrafish in vivo (Westfall et al., 2003; Vergara and Del Rio-Tsonis, 2009; Belgacem and Borodinsky, 2011; Stulberg et al., 2012; Das et al., 2016). In all cases, the control embryos were exposed to the same concentration of DMSO solvent used to dissolve the drugs. We assessed both the spatial patterns and the levels of sema3a and slit1 expression following inhibitor treatment by wholemount ISH and RT-qPCR, respectively.

By ISH, embryos treated with the $\mathrm{PLC}_{\gamma}$ inhibitor U73122 showed no obvious change when comparing the control treatment $(n=46$ brains, $N=4$; Fig. $4 B, n=39$ brains, $N=3$; Fig. $4 C)$ with that of $\mathrm{U} 73122(n=12$ brains, $N=3$; Fig. $4 D, n=11$ brains, $N=2$; Fig. $4 E$ ). Similarly, inhibition of the MAPK pathway produced no obvious qualitative differences in the expression of sema3a $(n=$ 11 brains, $N=2$; Fig. $4 F)$ and slit $1(n=11$ brains, $N=2$; Fig. 4G). Importantly, U0126 effectively blocked Mek activity in this in vivo assay, as assessed by Western blot analysis of the phosphorylated form of Erk in control and inhibitor-treated brains $(n=18$ brains and $N=3$ for both treated and control; Fig. 4H). We used RT-qPCR to verify the lack of change in the sema3a and slit1 ISH signal we observed. In agreement with the ISH data, no significant changes in sema3a $(p=0.61$; row k, Table 2$)$ and slit1 $(p$ $=0.99$; row I, Table 2) levels were observed by RT-qPCR with U0126 treatment $(n=23$ brains and $N=4$ for both U0126 and control; Fig. 4/). As a positive control for RT-qPCR we confirmed that sema3a and slit1 levels were decreased after $5 \mathrm{~h}$ of Fgfr inhibition by SU5402 (100 $\mu \mathrm{M})$, as we had observed previously by ISH (AtkinsonLeadbeater et al., 2010): SU5402 blocked Fgfr signaling, shown by decreased spry 1 ( $29 \%$ of control; $n=17$ brains and $N=3$ for both SU5402 and control, $p=0.049$; Fig. $4 \mathrm{~J}$; row $\mathrm{m}$, Table 2), and lowered sema3a and slit1 levels to $26 \%(p=0.015$; row $n$, Table 2$)$ and $32 \%(p=0.012$; row o, Table 2) of that of control, respectively. Importantly, however, mRNA levels of slit2, whose expression in the dorsal diencephalon we found previously to be Fgfindependent (Atkinson-Leadbeater et al., 2010), were not significantly affected by SU5402 $p=0.410$; row $p$, Table 2).

With PI3K pathway blockade by LY294002, however, there was visible reduction of signal for both sema3a $(n=$ 15/20 LY294002 brains, $N=3$; Fig. $4 B, K)$ and slit1 $(n=$ 11/15 LY294002 brains, $N=3$; Fig. 4C,L). Levels of sema3a in the forebrain were visibly lowered, while slit1 was reduced in the ventral telencephalon, hypothalamus, diencephalon, and adjacent to the pineal gland. Interestingly, ISH of slit1 expression in the ventral neural tube caudal to the midbrain was not noticeably different in LY294002-treated and control brains, suggesting a different mechanism of slit1 regulation in this brain region. When assessed quantitatively by RT-qPCR, LY294002 decreased sema3a and slit1 to $9.5 \%(p=0.002$; row $q$, 
A

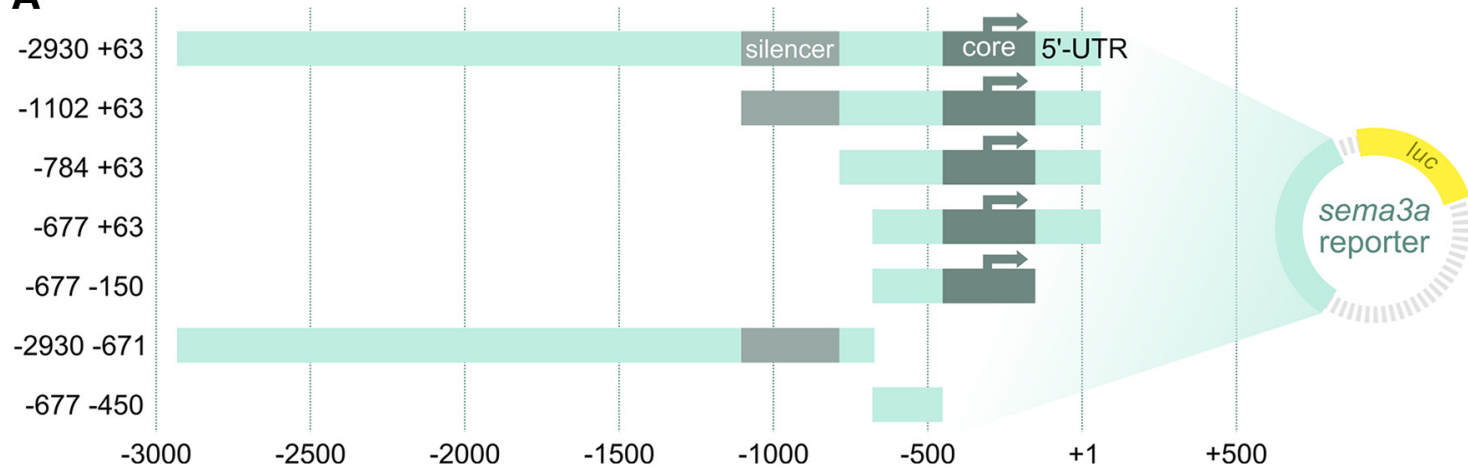

B

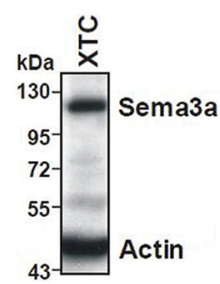

D

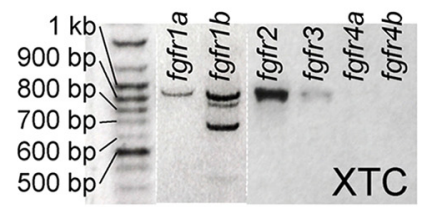

C

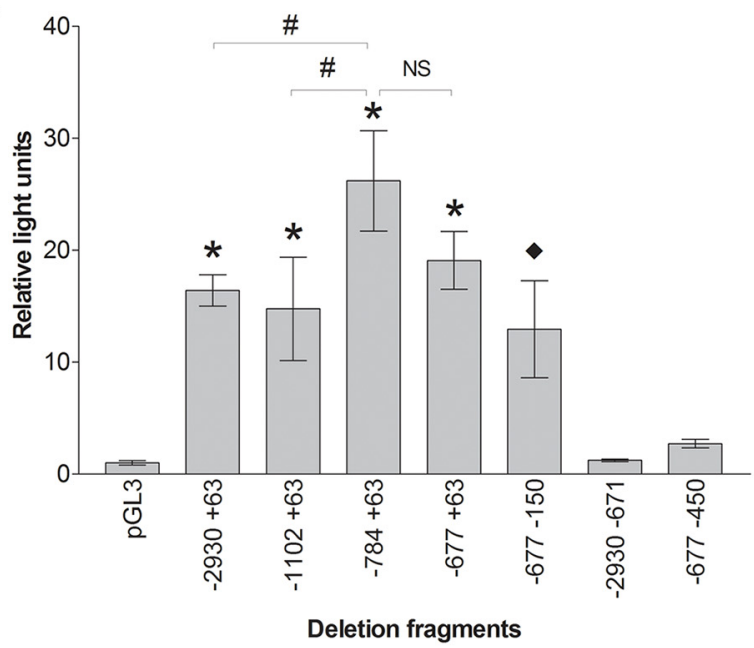

E

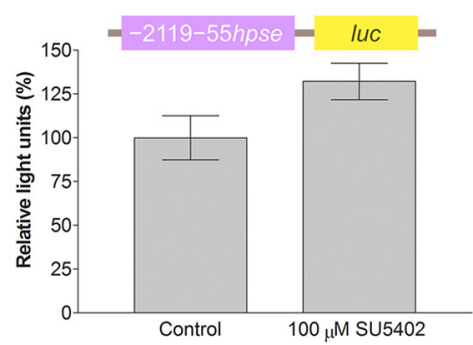

F

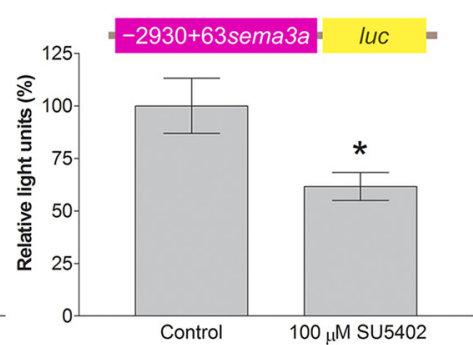

G

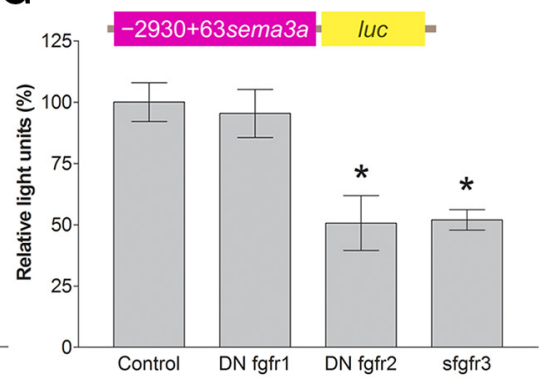

Figure 2. Characterization of the sema3a promoter. $\boldsymbol{A}$, Schematic of the sema3a deletion fragments inserted upstream of the firefly luciferase (IUC) gene in the pGL3 vector. B, XTC cells express endogenous Sema3a protein by Western blotting. $\boldsymbol{C}$, The reporter constructs of the sema3a promoter fragments and the Renilla luciferase construct were co-transfected into XTC cells. Luminescence corresponding to each deletion fragment was normalized to Renilla to account for transfection efficiency and expressed as relative light units compared to the promoterless $p G L 3$ basic vector. Each bar represents mean \pm SEM from $n=9$ wells, $N=3$. Statistical significance was determined by one-way ANOVA $(\alpha=0.05)$ and the following post hoc tests: ${ }^{\star} p<0.05$ and $* p<0.01$ by Dunnett's multiple comparison test versus the control ( $p G L 3$ vector) and $\# p<0.05$ by Bonferroni's multiple comparison test between selected deletion fragments. $\boldsymbol{D}$, Expression of fgfr genes in XTC cells, shown by RT-PCR. XTC cells express all the fgfr genes (as verified by sequencing of amplicons) except for fgfr $4 a / b$. Of note, the fgfr $1 b$ amplicons smaller than 900 bp may be alternatively spliced variants (Friesel and Dawid, 1991). $\boldsymbol{E}, \boldsymbol{F}$, The heparanase promoter construct (E, $n=12$ wells, $N=2$ for both bars), $-2119-55$ hpse::/uc, and the $-2930+63$ sema3a::/uc construct $(\boldsymbol{F}, n=12$ wells, $N=3$ for both bars) were co-transfected into XTC cells in 96-well plates with the Renilla luciferase plasmid and treated with $100 \mu \mathrm{M}$ SU5402. Bars reflect mean $\pm \mathrm{SEM} ; * p<0.05$, compared to DMSO control, two-tailed Student's $t$ test. G, $-2930+63$ sema3a::luc was co-transfected with truncated fgfrs in XTC cells $(N=7$ for all bars; control $n=30$ wells, DN fgfr $1 n=16$ wells, DN fgfr2 $n=12$ wells, sfgfr3 $n=14$ wells). Bars reflect mean \pm SEM; $* p<0.05$ by ANOVA and Bonferroni post hoc test compared to pCS2-GFP transfection as control. 

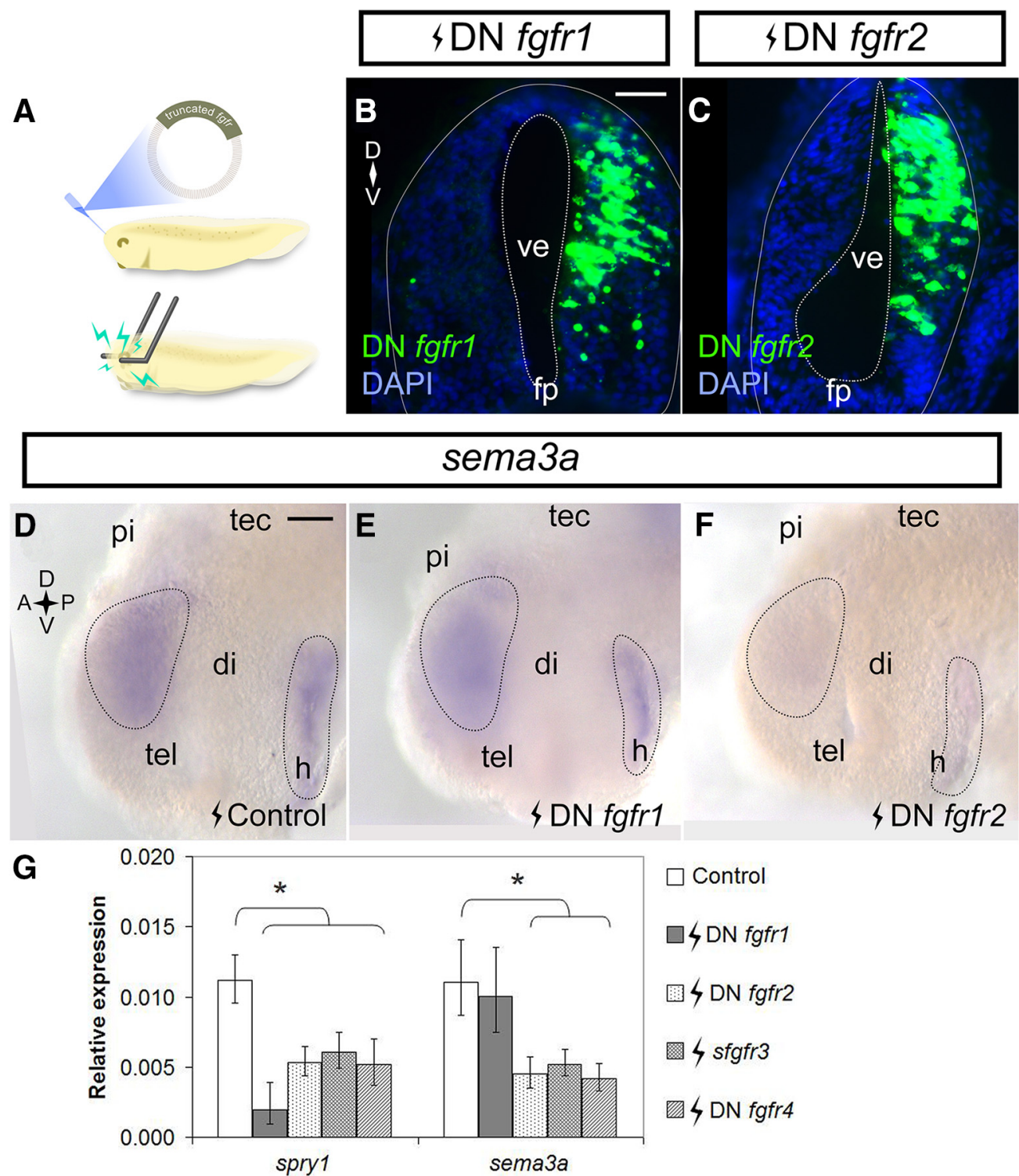

Figure 3. Fgfr2-4 inhibition downregulates sema3a in the forebrain. A, A cartoon of stage $27 / 28$ forebrain electroporation. $\boldsymbol{B}$, $\boldsymbol{C}$, Embryos were electroporated with pCS2-DNfgfr1 $(\boldsymbol{B})$ and pCS108-DNfgfr2 $(\boldsymbol{C})$ and processed in transverse cryostat sections by FISH for fgfr $1 / 2$ at stage 32 to confirm transgene expression. $\boldsymbol{D}-\boldsymbol{F}$, Embryos were electroporated with control $p C S 2-G F P(\boldsymbol{D}, n=37$ brains, $N=3$ ), pCS2-DNfgfr1 ( $\boldsymbol{E}, n=20$ brains, $N=3)$, or pCS108-DNfgfr2 $(\boldsymbol{F}, n=16 / 24$ brains had decreased expression, $N=3)$ and processed for sema3a expression by wholemount ISH at stage 32. G, sema3a mRNA levels in brains electroporated with truncated fgfrs was measured by RT-qPCR. spry 1 was a readout of Fgfr inhibition. Bars represent mean $\pm \mathrm{SEM}$ for control $(n=32, N=4)$, DN fgfr1 $(n=24, N=4)$, DN fgfr2 $(n=20, N=3)$, sfgfr3 $(n=30, N=4)$, and DN fgfr4 $(n=31, N=4) ; * p<0.05$ statistical significance versus the control was determined using the REST algorithm. Scale bars, $50 \mu \mathrm{m}$. di, diencephalon; fp, floor plate; $h$, hypothalamus; pi, pineal gland; tec, optic tectum; tel, telencephalon; ve, ventricle.

Table 2) and $17 \%(p=0.022$; row $r$, Table 2$)$ of control levels, respectively, while slit2 ( $p=0.255$; row s, Table 2$)$ was unchanged $(n=11$ brains and $N=2$ for both LY294002 and control; Fig. 4M). Together, these data suggest that the PI3K pathway positively regulates sema3a and slit1 expression in the forebrain. Interestingly, spry1 was unaffected by $\mathrm{U} 0126(p=0.454$; row $t$, Table 2), but downregulated by SU5402 ( $p=0.049$; row $u$, Table 2) and LY294002 ( $p=0.041$; row v, Table 2), suggesting that Spry1 may be a feedback regulator that functions specifically downstream of PI3K. In agreement,
LY294002 downregulates Spry1 expression in bovine granulosa cells (Jiang et al., 2011; Jiang and Price, 2012). Therefore, the ISH and RT-qPCR data support the idea that Fgfrs promote sema3a and slit1 transcription in the forebrain via PI3K signaling. The speed of the dysregulation with LY294002 argues against cell death or proliferation as explanations for the decreased guidance cue expression.

To support the idea that the PI3K pathway acts downstream of Fgfr signaling to maintain sema3a and slit1 mRNA levels, we first assessed PI3K pathway activation 
A

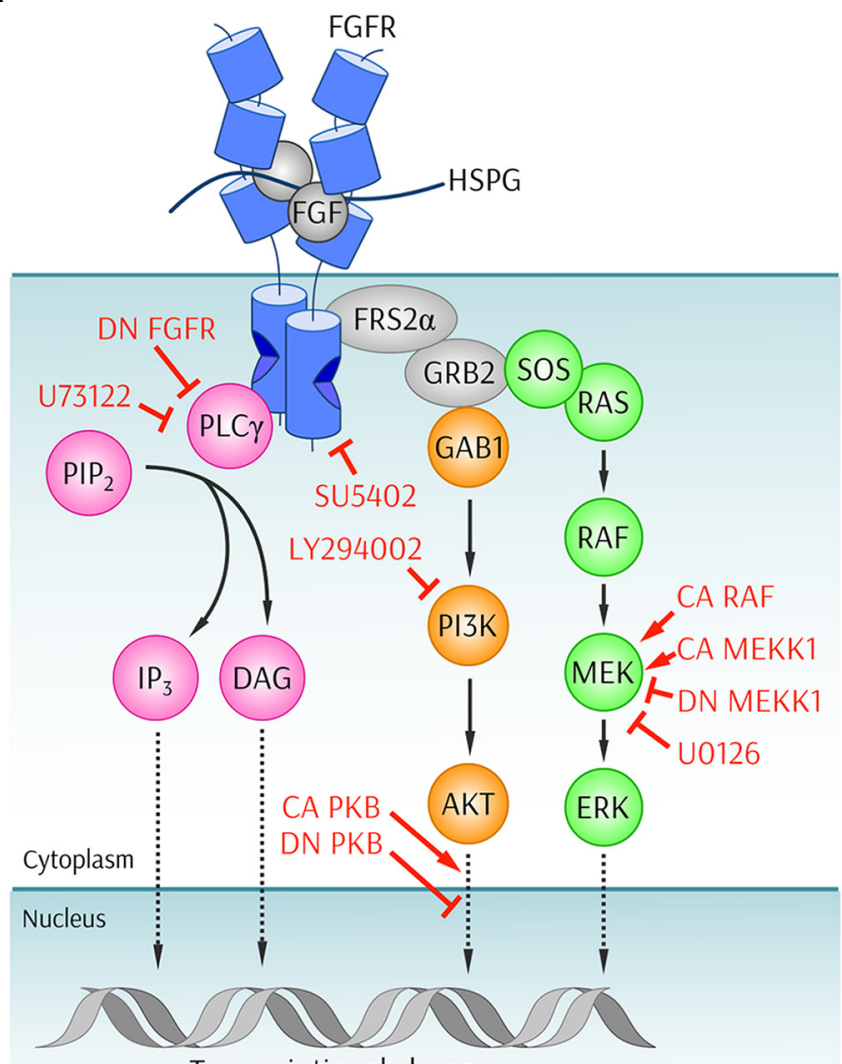

Transcriptional changes

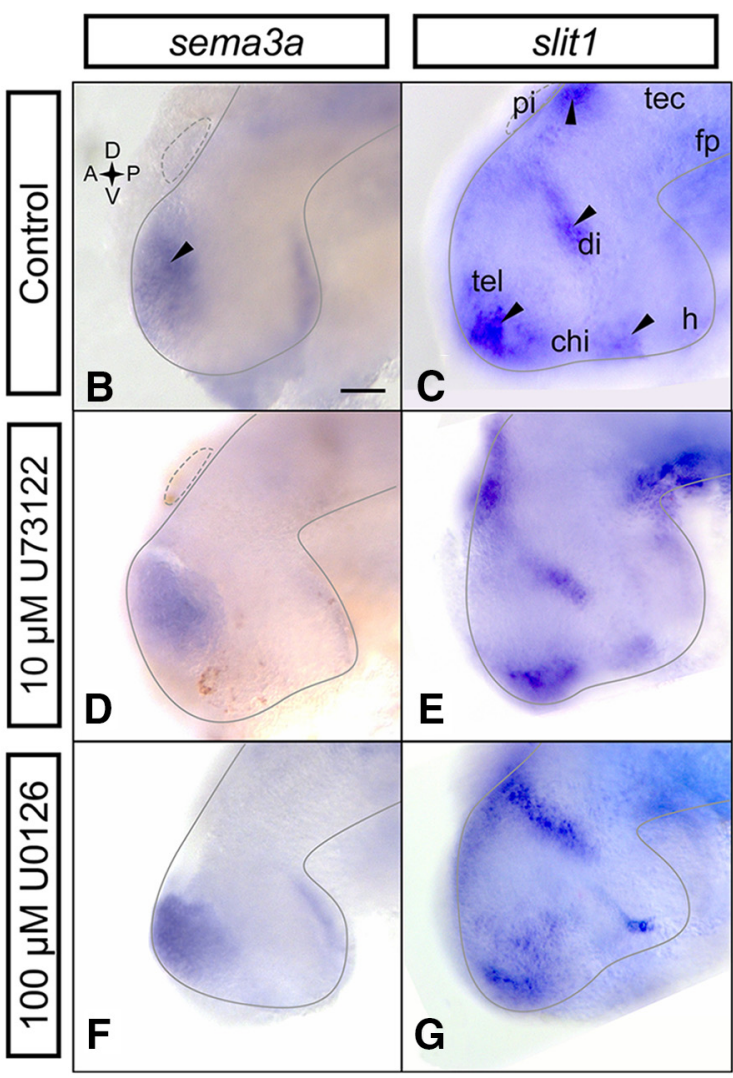

H

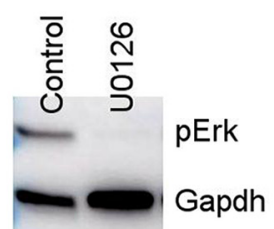

I
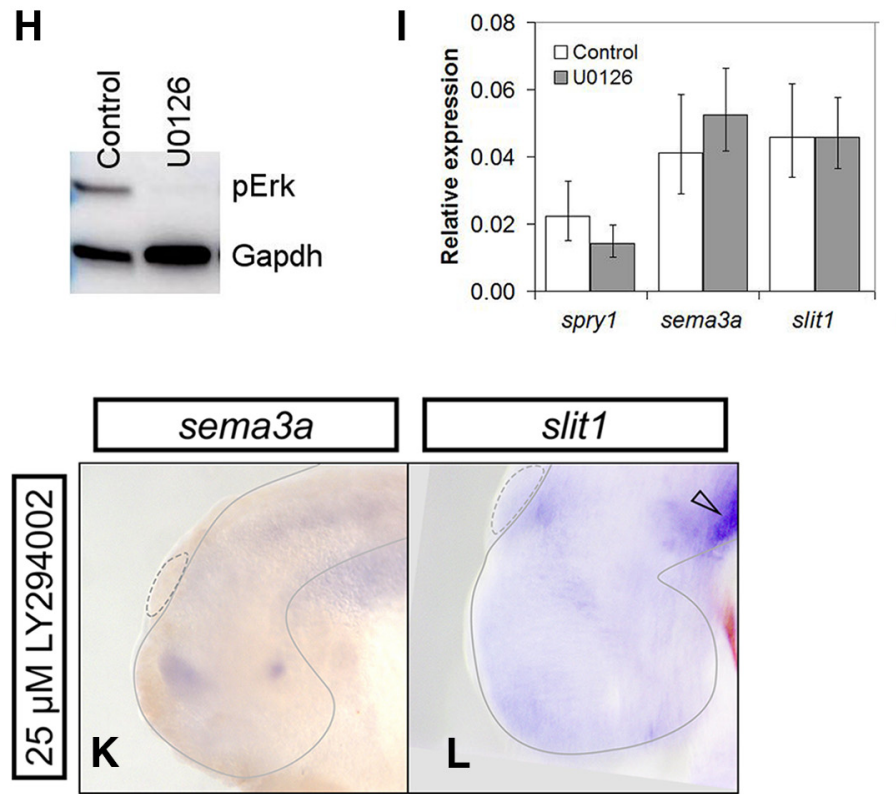
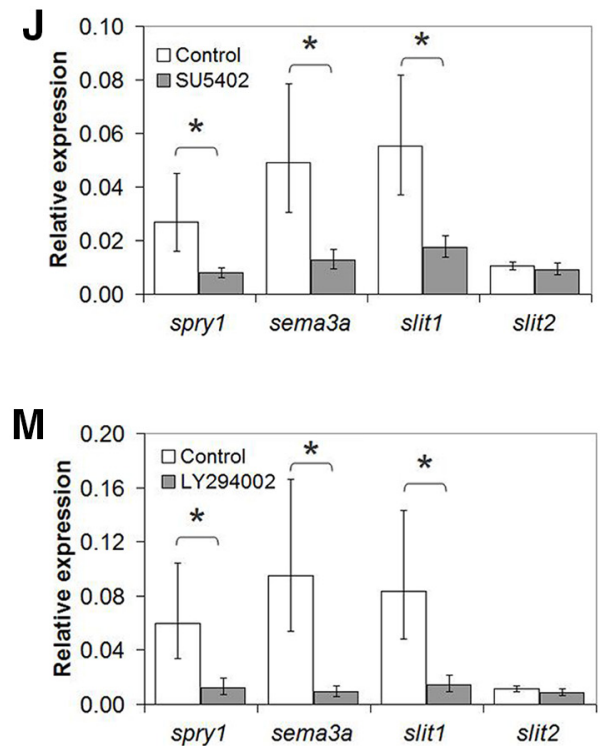

Figure 4. PI3K inhibition decreases both sema3a and slit1 expression in the forebrain. $\boldsymbol{A}$, The canonical sequence of Fgf signaling intermediates is indicated by solid arrows. Dotted arrows show further signaling through downstream factors (Rodriguez-Viciana et al., 1994; Zimmermann and Moelling, 1999; Jiminez et al., 2002; Nakayama et al., 2008; Aksamitiene et al., 2012; Mendoza et al., 2011; Chen et al., 2012; Selvaraj et al., 2014). The activated complex of HSPG-FGF-FGFR dimerizes to phosphorylate the FGFR intracellular tails and recruits FRS2 $\alpha$, GRB2, GAB1, SOS, and RAS (Rubinfeld and Seger, 2004; Lemmon and Schlessinger, 2010). GAB1 activates the PI3K-AKT relay (orange). RAS activates the RAF-MEK-ERK cascade, i.e., MAPK signaling (green). PLC $\gamma$ can directly bind to the phosphorylated FGFR to hydrolyze PIP to $_{2} \mathrm{P}_{3}$ and DAG (magenta). The inhibitors against FGFRs, MEK, PI3K, and PLC $\gamma$ are SU5402, U0126, LY294002, and U73122, respectively. CA RAF and CA MEKK1 were used to overactivate the ERK MAPK 
continued

pathway. DN MEKK1 was used to inhibit the MAPK pathway. The CA and DN PKB (AKT) constructs were used for gain and loss of function of AKT signaling, respectively. Wild-type PLC $\gamma$ was overexpressed to increase PLC $\gamma$ signaling, and the PLC $\gamma$-deficient DN FGFR was used to inhibit PLC $\gamma$ activation. $\boldsymbol{B}-\mathbf{G}$. Stage 32 brains were treated with control $(\boldsymbol{B}, n=46$ brains, $N=4 ; \boldsymbol{C}, n=39$ brains, $N=3$ ) or $10 \mu \mathrm{M}$ U73122 solution and processed by wholemount ISH for sema3a $(\boldsymbol{D}, n=12$ brains, $N=3)$ and slit1 (E, $n=11$ brains, $N=2$ ). Black arrowheads indicate the sema3a and slit1 domains of interest. Mek inhibition with $100 \mu \mathrm{M}$ U0126 treatment did not visibly decrease sema3a $(\boldsymbol{F}, n=11$ brains, $N=2$ ) or slit1 ( $\boldsymbol{G}, n=11$ brains, $N=2$ ) expression by wholemount ISH. $\boldsymbol{H}$, Phospho-Erk (pErk) knockdown in U0126-treated forebrains confirmed by Western blot analysis $(n=18$ brains and $N=3$ for both treated and control). I, J, RT-qPCR of sema3a and slit1 forebrain mRNA following treatment with U0126 (I, $n=23$ brains and $N=4$ for both U0126 and control) and $100 \mu \mathrm{M}$ SU5402 ( $\boldsymbol{J}, n=17$ brains and $N=3$ for both SU5402 and control). $\boldsymbol{K}-\boldsymbol{M}$, PI3K inhibition with $25 \mu \mathrm{M}$ LY294002 treatment decreased sema3a $(K, n=15 / 20$ LY294002 brains had decreased expression vs the control in $\boldsymbol{B}, N=3)$ and slit1 ( $\boldsymbol{L}, n=11 / 15$ LY294002 brains had decreased expression vs the control in $\boldsymbol{C}, N=2$ ) expression by ISH. slit1 expression in the floor plate $(\boldsymbol{L}$, unfilled arrowhead) was unaffected by LY294002 treatment. $\boldsymbol{M}$, RT-qPCR for sema3a and slit1 mRNA with LY294002 treatment ( $n=11$ brains and $N=2$ for both LY294002 and control). In all RT-qPCR data, bars represent the mean \pm SEM; $* p<0.05$ using the REST algorithm for statistical significance. Scale bar, $50 \mu \mathrm{m}$. chi, optic chiasm; di, diencephalon; fp, floor plate; h, hypothalamus; pi, pineal gland; tec, optic tectum; tel, telencephalon.

in the developing forebrain with Fgfr inhibition. Brains were exposed at stage 33/34 to the Fgfr inhibitor SU5402 $(100 \mu \mathrm{M})$ and were collected $5 \mathrm{~h}$ later, and processed by Western blot analysis for the phosphorylated form of Akt (pAkt), a readout of PI3K pathway activation. pAkt levels were downregulated with SU5402 as compared to control (data not shown), indicating that Fgfr forebrain signaling promotes PI3K pathway activity.

\section{Molecular blockade of PI3K signaling inhibits both sema3a and slit1 expression}

To confirm the pharmacological inhibition data, we turned to a molecular approach to disrupt the candidate pathways through electroporation of plasmids into the stage $27 / 28$ brain that allowed for the gain and loss of function of specific signaling intermediates (Fig. 4A). Forebrain sema3a and slit1 mRNA levels were assessed by RT-qPCR $24 \mathrm{~h}$ after electroporation. In agreement with the U73122 data, molecular manipulation of the PLC $\gamma$ pathway did not affect sema3a and slit1 expression. A plasmid expressing PLC $\gamma$ did not affect sema3a $(p=$ 0.520; row w, Table 2) and slit1 ( $p=0.594$; row $x$, Table 2) levels; however, PLC $\gamma$ overexpression increased spry 1 levels to almost twice ( $181 \%, p=0.016$; row y, Table 2$)$ that of control, suggesting strong Fgf signal activation (PLC $\gamma n=32$ brains and $N=4$ vs control $n=31$ brains and $N=4$; Fig. 5A). Furthermore, preventing PLC $\gamma$ activation downstream of Fgfr1, by expression of the phRlucN1-FGFR1Y766F construct that encodes for human FGFR1 mutated at Tyr766 to prevent binding to PLC $\gamma$ (Browaeys-Poly et al., 2010), failed to change sema3a ( $p$ $=0.351$; row $z$, Table 2$)$ and slit1 $(p=0.654$; row aa, Table 2) levels (FGFR1Y766F $n=22$ brains and $N=3$ vs control $n=24$ brains and $N=3$; Fig. $5 B$ ).

Similarly, in agreement with the MAPK U0126 data, molecular blockade of the pathway by electroporation with DN MEKK1 (Fig. 5C), pCS2-MEKK1-KM (Ben Messaoud et al., 2015), produced no change in sema3a ( $p=$ 0.478 ; row ab, Table 2) or slit1 ( $p=0.372$; row ac, Table 2) expression (MEKK1-KM $n=30$ brains and $N=4$ vs control $n=31$ brains and $N=4$; Fig. $5 D$ ). Interestingly, the MAPK cascade appears to be sufficient to control sema3a and slit1 transcription. Electroporation of consti- tutively active (CA) MEKK1 (Fig. 5E), pCS2-MEKK1+ (Ben Messaoud et al., 2015), downregulated sema3a and slit1 mRNA to $80.7 \%$ ( $p=0.023$; row ad, Table 2 ) and $76.1 \%$ $(p=0.041$; row ae, Table 2$)$ of control electroporation (MEKK1+ $n=28$ brains and $N=4$ vs control $n=31$ brains and $N=4$; Fig. 5F). Likewise, CA B-RAF (pCS108BRAFV600E; Boehm et al., 2007; Tachibana et al., 2016) electroporation (Fig. 5G) decreased sema3a and slit1 expression to $76.2 \%$ ( $p=0.008$; row af, Table 2$)$ and $65.9 \%$ $(p<0.001$; row ag, Table 2) of control electroporation, respectively (BRAFV600E $n=32$ brains and $N=4$ vs control $n=31$ brains and $N=4$; Fig. $5 H$ ). Of note, these changes in gene expression were likely underestimated in that only half of the brain generally expressed the transgenes after electroporation. Thus, MAPK activation is sufficient but not necessary to control sema3a and slit1, and in a manner distinctive of Fgfr-PI3K signaling, as spry1 expression was unaffected. In summary, the molecular data confirm that neither the MAPK nor PLC $\gamma$ pathways are required to maintain sema3a and slit1 expression.

In contrast, molecular modulation of PI3K-Akt signaling affected expression of these two guidance cues. By RTqPCR, DN AKT electroporation (Fig. 6A), ${ }^{T 308 A / S 473 A} P K B$ (Palmada et al., 2005) reduced sema3a and slit1 to $66.7 \%$ $(p=0.022$; row ah, Table 2$)$ and $74.4 \%(p=0.007$; row ai, Table 2) of control levels, respectively $\left({ }^{T 308 A / S 473 A}\right.$ PKB $n=$ 21 brains and $N=3$ vs control $n=23$ brains and $N=3$; Fig. 6B). The RT-qPCR data for Akt loss of function is consistent with the ISH and RT-qPCR data showing that PI3K inhibition reduces sema3a and slit1 levels. In contrast, CA AKT electroporation (Fig. 6C), ${ }^{T 308 D / S 473 D} P K B$, AKT with activating phosphomimetic mutations (Palmada et al., 2005), upregulated sema3a and slit 1 in the forebrain to $158 \%(p=0.001$; row aj, Table 2$)$ and $131 \%(p=0.043$; row ak, Table 2) of control, respectively ${ }^{T 308 D / S 473 D} P K B n=40$ brains and $N=5$ vs control $n=39$ brains and $N=5$; Fig. $6 D$ ). Similarly, AKT-myr electroporation (Fig. 6E), a myristoylated CA AKT (Jin et al., 2015), upregulated sema3a and slit 1 to $175 \%(p=0.001$; row al, Table 2$)$ and $123 \%$ $(p=0.019$; row am, Table 2$)$ of control, respectively $(n=$ 22 brains and $N=3$ vs control $n=24$ brains and $N=3$; Fig. 6F). Importantly, modulation of Akt activity resulted in corresponding changes in the levels of the Fgfr pathway 
A

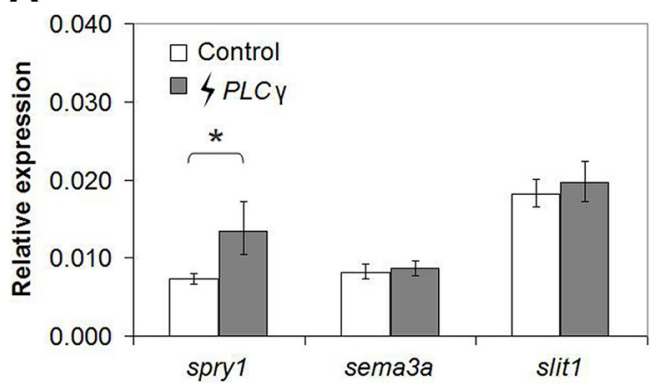

B

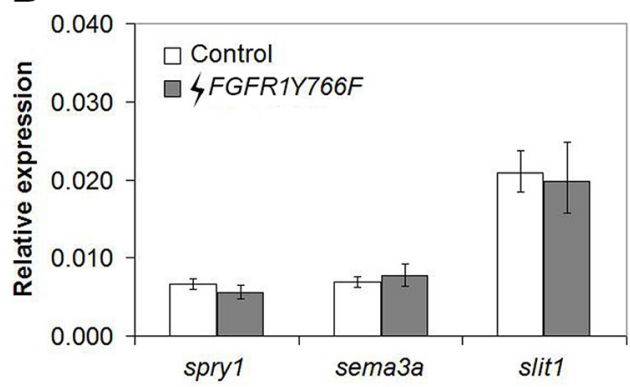

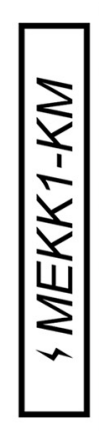
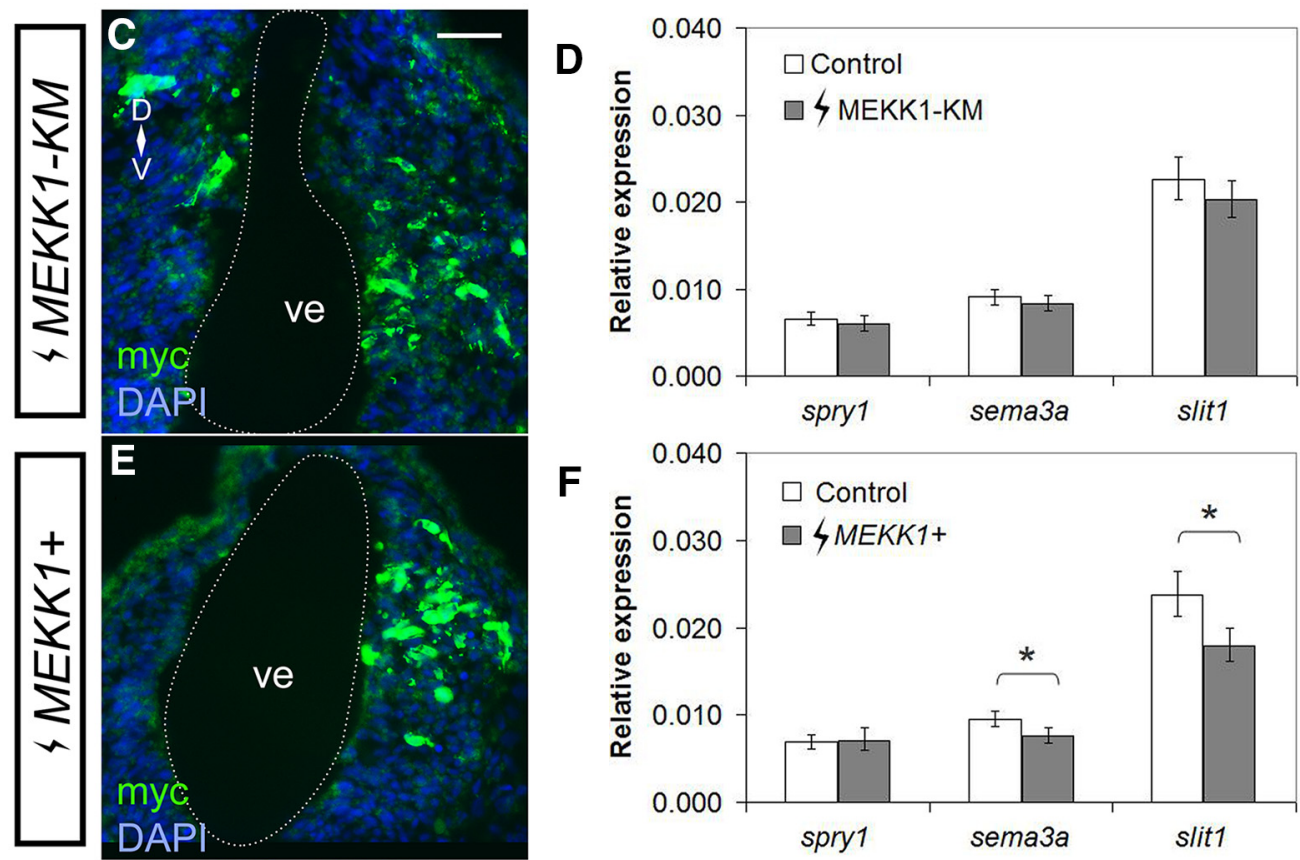

$\mathbf{F}$
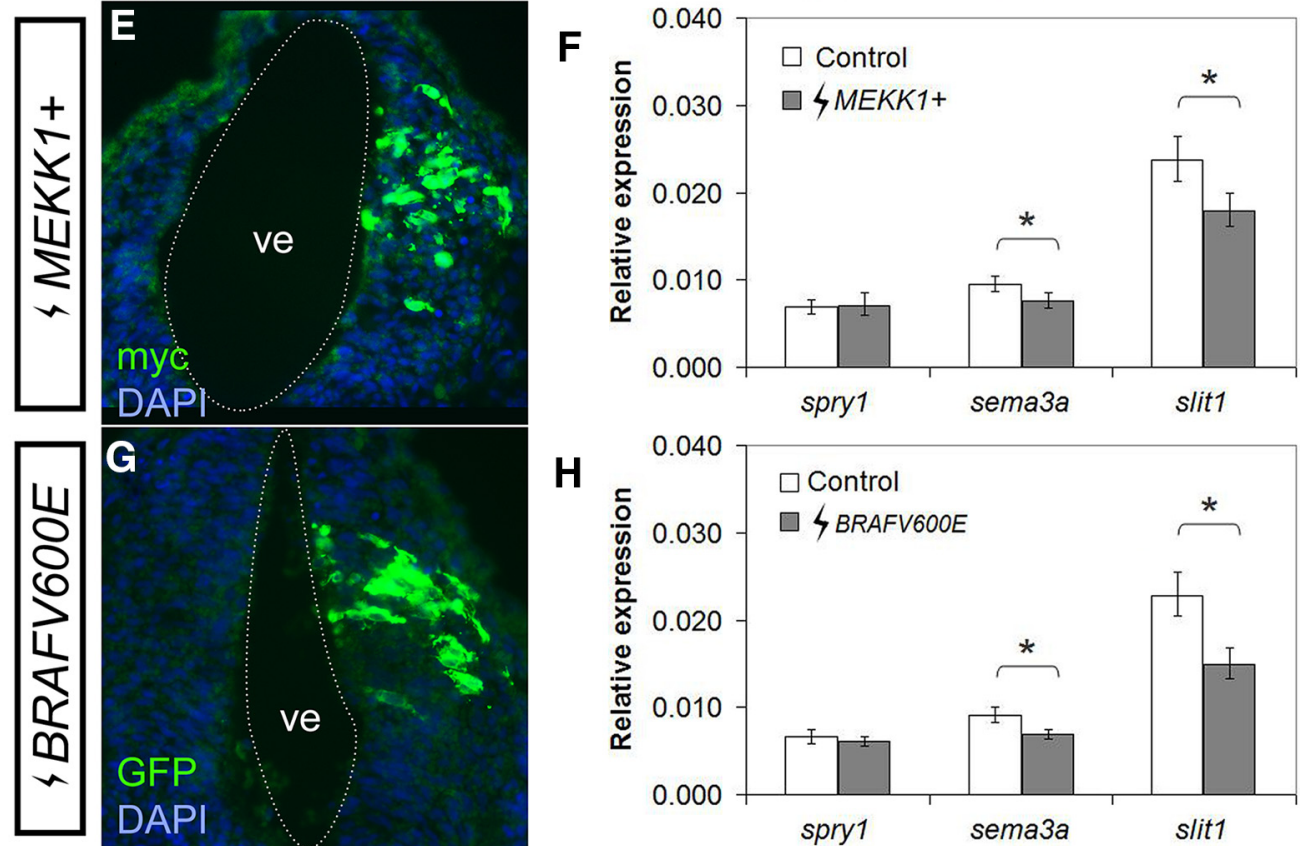

Figure 5. Activation of the MAPK pathway is sufficient to inhibit both sema3a and slit1 expression in the forebrain. Constructs for Fgfr downstream signaling intermediates were electroporated into the forebrain of stage 27/28 embryos, and spry 1 , sema3a, and slit1 expression in the forebrain were quantified by RT-qPCR $24 \mathrm{~h}$ after electroporation (stage 32). $\boldsymbol{A}, \boldsymbol{B}$, RT-qPCR when PLC $\gamma$ signaling was augmented by $P E Y F P-P L C \gamma(\boldsymbol{A}, P L C \gamma n=32$ brains, $N=4$ vs control $n=31, N=4)$, and inhibited with the PLC $\gamma$-deficient FGFR1, phRluc-N1-FGFR1Y766F (B, FGFR1Y766F $n=22$ brains, $N=3$ vs control $n=24, N=3$ ). C-G, The MAPK pathway was inhibited with a DN MEKK1, pCS2-MEKK1-KM, and activated with either pCS2-MEKK1+ or pCS108-BRAFV600E. Immunohistochemistry on transverse sections of the forebrain against myc $(\boldsymbol{C}, \boldsymbol{E})$ and GFP $(\boldsymbol{G})$ tags confirm successful electroporations. Changes in gene expression was quantified by RT-qPCR after electroporation with $M E K K 1-K M(\boldsymbol{D}, n=30, N=4), M E K K 1+(\boldsymbol{F}, n=28, N=$ 4), and BRAFV600E $(\boldsymbol{H}, n=32, N=4)$ and compared to control pCS2-GFP electroporation $(\boldsymbol{D}, \boldsymbol{F}, \boldsymbol{H}, n=31, N=4)$. Bars represent the mean \pm SEM; $* p<0.05$ using the REST algorithm for statistical significance. Scale bar, $50 \mu \mathrm{m}$. ve, ventricle.

feedback regulator, spry 1 , whereby CA $A K T$ increased transcription (318\%, $p=0.004$; row an, Table 2) and DN $A K T$ repressed transcription $(67.8 \%, p=0.041$; row ao,
Table 2). The fact that changes in this known Fgfr downstream effector are observed with PI3K-Akt manipulation both molecularly and pharmacologically, argues that Fgfr 

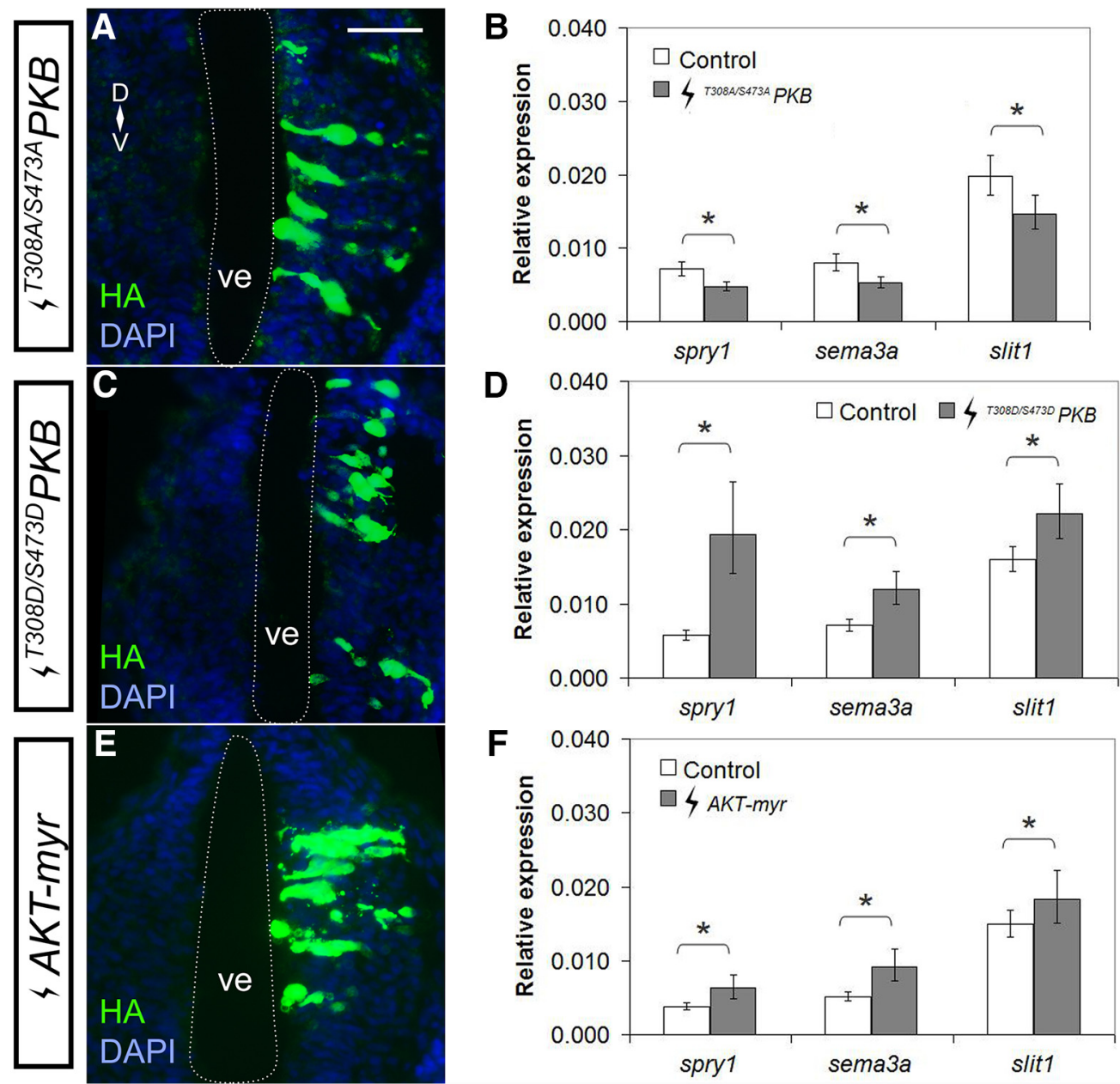

Figure 6. Akt signaling positively regulates sema3a and slit1 in the forebrain. Plasmids encoding DN $A K T(\boldsymbol{A}, \boldsymbol{B})^{T 308 A / S 473 A} P K B$, and $\mathrm{CA} A K T(\boldsymbol{C}, \boldsymbol{D})^{T 308 D / S 473 D} P K B$, and $(\boldsymbol{E}, \boldsymbol{F}) A K T$-myr were electroporated into the forebrain of stage $27 / 28$ embryos. $\boldsymbol{A}, \boldsymbol{C}, \boldsymbol{E}$, Forebrain transgene expression was assessed by immunohistochemistry for HA-tagged AKT mutants. $\boldsymbol{B}$, $\boldsymbol{D}, \boldsymbol{F}$, spry1, sema3a, and slit1 expression in the forebrain $24 \mathrm{~h}$ after electroporation was quantified by RT-qPCR for $(\boldsymbol{B}){ }^{T 308 A / S 473 A} P K B$ ( $n=21, N=3$ vs control $n$ $=23, N=3),(\boldsymbol{D})^{T 308 D / S 473 D} \mathrm{PKB}(n=40, N=5 \mathrm{vs}$ control $n=39, N=5)$, and $(\boldsymbol{F})$ AKT-myr $(n=22, N=3$ vs control $n=24, N=$ $3)$, where the control electroporation was $p C S 2-G F P$. Bars represent the mean $\pm \mathrm{SEM} ; * p<0.05$ using the REST algorithm for statistical significance. Scale bar, $50 \mu \mathrm{m}$. ve, ventricle.

signaling regulates sema3a and slit1 through the PI3K-Akt pathway.

\section{Inhibition of PI3K signaling in vivo disrupts RGC axon guidance at the mid-diencephalic turn}

Previously, we found that downregulation of sema3a and slit1 expression, directly through brain electroporation of antisense oligonucleotides or indirectly via pharmacological inhibition of Fgf signaling with SU5402, caused RGC axons to stall in the mid-diencephalon and fail to move on toward their dorsal midbrain target, the optic tectum (Atkinson-Leadbeater et al., 2010). If Fgf signaling works through the PI3K pathway to help maintain sema3a and slit1 guidance cues we expected a similar axon "stall" phenotype with PI3K pathway inhibition by application of LY94002 $(20-25 \mu \mathrm{M})$ in vivo in the developing forebrain neuroepithelium. We performed the exposed brain preparation at stage 33/34, when the first RGC axons from the right eye enter the contralateral ventral diencephalon before extending dorsally through the diencephalon. Axon guidance was assessed by anterogradely labeling RGC axons by HRP at stage 40 , when normally the vast majority of RGC axons have grown into and innervated the optic tectum (Holt, 1989).

As expected, in control DMSO brains RGC axons targeted the optic tectum ( $n=16$ brains, $N=3$; Fig. $7 A)$. In contrast, when brains were exposed to LY94002 $6 \mathrm{~h}$ before the first RGC axons reach the mid-diencephalon, many of the optic tracts had significant numbers of axons that stalled in the mid-diencephalon and failed to navigate to the optic tectum, similar to what we observed previously with Fgf signaling inhibition $(n=33$ brains, $N=3$; Fig. 7B,C; Atkinson-Leadbeater et al., 2010). Of note, given the likelihood that the PI3K pathway has additional roles in the formation of the optic projection, the LY294002 phenotype was not identical to that produced with the Fgfr inhibitor; for instance, the LY294002 optic tract appeared defasciculated (Fig. 7C), a phenotype not observed with SU5402. 

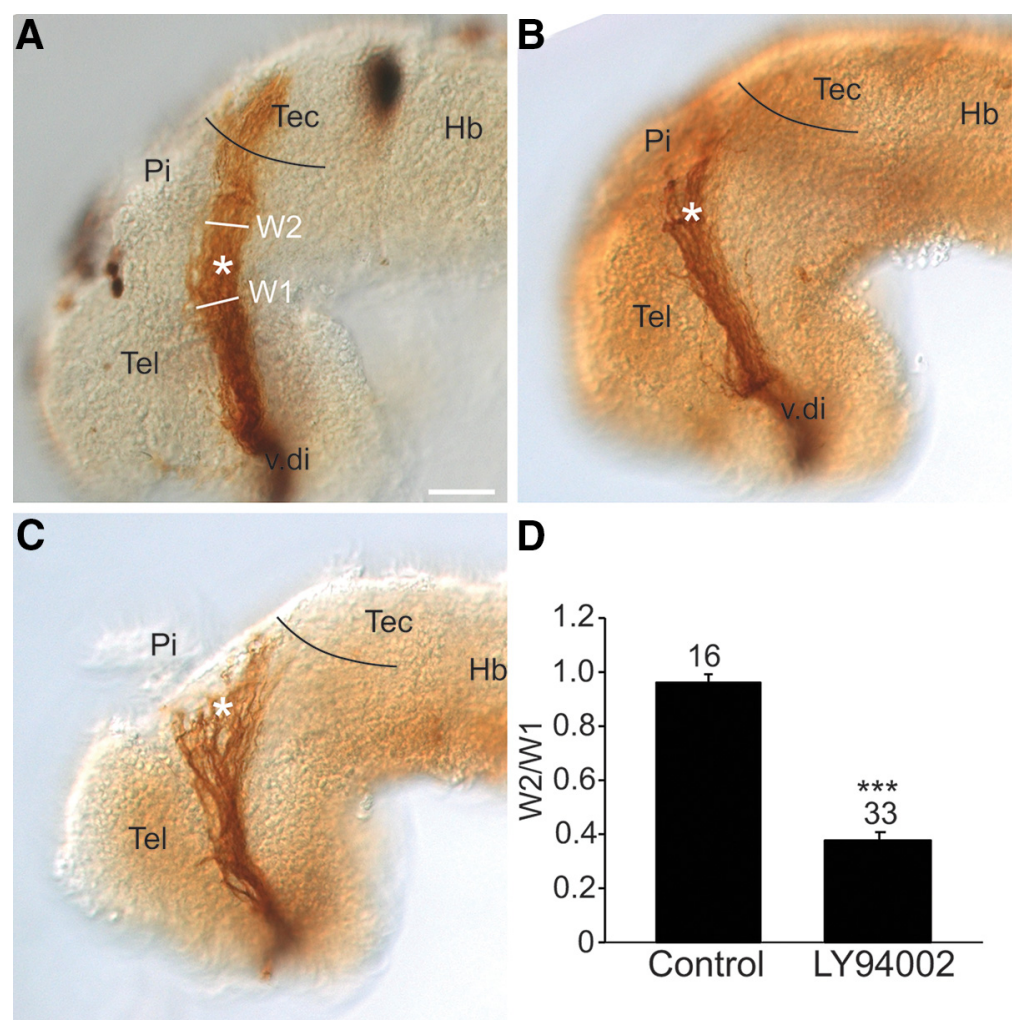

D

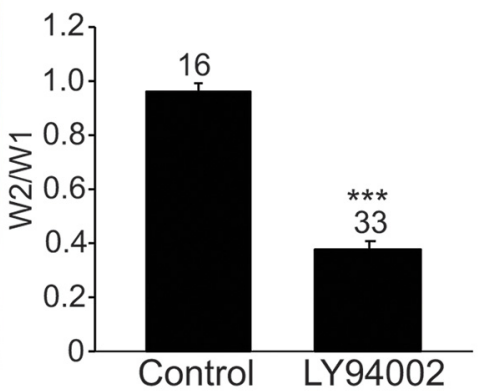

Figure 7. PI3K signaling regulates the behavior of RGC axons at the mid-diencephalic turn. Lateral views of stage 40 wholemount brains with HRP-labeled optic tracts that were exposed at stage 33/34 either to $(\boldsymbol{A})$ control DMSO solution $(n=16$ brains, $N=3)$ or $(\boldsymbol{B}, \boldsymbol{C}) 20 \mu \mathrm{M}$ LY294002 ( $n=33$ brains, $N=3$ ). Black line represents the approximate anterior border of the optic tectum, and the asterisk the location of the mid-diencephalic turn guidance choice point. $\boldsymbol{D}$, The RGC axon stall phenotype was quantified by representing the width of the optic tract post-mid-diencephalic turn (W2) as a ratio (W2/W1) to the width of the optic tract (W1) at the mid-diencephalic turn. On each bar is the number of individual brains pooled from 3 independent experiments. Bars represent the mean \pm SEM; $* * * p<0.001$ using the unpaired Student's $t$ test. Scale bar, $50 \mu \mathrm{m}$. Hb, hindbrain; Pi, pineal gland; Tec, tectum; Tel, telencephalon; v.di, ventral diencephalon.

We quantified the stall phenotype by measuring the width of the optic tract at the mid-diencephalic turn, and then the widest portion of the optic tract distal to the turn, and represented these widths as a ratio. While in control embryos the tract was generally the same width at the two locations, in PI3K inhibitor-treated brains, the optic tract was significantly ( $p<0.001$; row ap, Table 2 ) narrower post-turn, reflecting the fall-out of axons at the middiencephalon (Fig. 7D). These data support the idea that PI3K works downstream of Fgfrs to help maintain the expression of Sema3a and Slit1, two repellents key for controlling the trajectories of RGC axons at the middiencephalic choice point. We propose that guidance of RGC axons at the mid-diencephalon depends on PI3K signaling in neuroepithelial cells maintaining Sema3a/Slit1 levels, and not PI3K signaling in the RGC growth cones themselves. In support, similar to what was observed with LY294002 brain exposures, inhibition of PI3K signaling only in brain neuroepithelial cells and not RGC axons, via electroporation of DN $A K T$ into stage 28 brains, produced an HRP-labeled optic projection that unlike GFP controls was significantly narrower after the mid-diencephalic turn $\left(p<0.0001\right.$; row aq, Table 2, ${ }^{\text {T308A/S473A } P K B ~} n=16$ brains and $N=3$ vs control $n=16$ brains and $N=3$ ). Of note, a prior pharmacological screen we performed failed to identify a role for the PI3K pathway in guiding RGC axons within the diencephalon (Webber et al., 2005). We feel this discrepancy reflects the nature of the simplified read-outs in the initial screen of mis-routing of axons from the optic tract and failure of optic tectum innervation. The "axon stalling" in the mid-diencephalon we characterized for the first time with Fgfr inhibition (Atkinson-Leadbeater et al., 2010), and observed here with PI3K inhibition, is often partial in nature, where some axons escape and go on to innervate the optic tectum. At the time of the screen, we would have classified these "partial stall" optic tracts as normal with regards to "guidance," because axons did reach the optic tectum and misrouting of axons from the optic tract was not observed. Thus, the PI3K inhibitor data provide additional support that Fgf signaling works through the PI3K pathway to maintain expression of guidance cues important for guidance of RGC axons in the diencephalon.

\section{Discussion}

Guidance cues direct the trajectory of growing axons so that proper synaptic connections can be made. While families of guidance cues have been identified, their transcriptional regulation has been largely unexplored nor has the co-regulation of two distinct guidance cues at the 
same axon choice point been considered. Previously, we found that guidance of the axons of the optic tract relies on repellents Sema3a and Slit1 expressed in the forebrain (Atkinson-Leadbeater et al., 2010). Building on our prior work (Yang et al., 2018), here we find that sema3a and slit1 genes are expressed in essentially non-overlapping domains of the forebrain, and are regulated by distinct Fgfrs. Despite these differences, our data support the idea that transactivation of sema3a and slit1 converges along the PI3K-Akt pathway. Thus, we identify for the first time in vivo the extracellular receptors and signal transduction pathway that regulate two key guidance cues at a single brain decision point, and suggest a common intrinsic mechanism.

Although sema3a and slit1 both depend on Fgf signaling for expression, and together repel RGC axons at the same mid-diencephalic guidance choice point (AtkinsonLeadbeater et al., 2010), our data argue that distinct Fgfrs control the expression of the two genes. Fgfr2-4 are key regulators of sema3a transcription, while Fgfr1 promotes slit1 expression (Yang et al., 2018). One possibility is that the choice of receptors simply reflects the differential expression of fgfrs in separate cell populations. slit1 mRNA is largely present in cells at the pial surface of the brain, which are likely post-mitotic, though it does overlap significantly with fgfr1 in ventricular progenitors in the anterior forebrain, and in the floor plate more caudally (Yang et al., 2018). In contrast, sema3a mRNA is present within the progenitor ventricular zone. Thus, the distinct sema3a/slit1 cell populations may express different Fgfr isoforms to respond to the specific Fgfs available locally. Two pieces of data argue against this possibility. First, fgfr 1 overlaps extensively with both sema3a and slit1, yet specific inhibition of Fgfr1 only reduces slit1 mRNA levels. Second, the forebrain data are consistent with those from cell culture where slit1 is regulated by Fgfr1 (Yang et al., 2018) and sema3a by Fgfr2/3. These data argue that distinct Fgfrs control the expression of specific guidance genes. The retinal expression data support this idea, in that only fgfr1 shows extensive overlap with that of slit 1 in differentiating RGCs, whereas mRNA for all four receptors is present in the sema3a-expressing cells of the lens (data not shown). Thus, while multiple Fgfrs appear to work together to promote sema3a expression, only Fgfr1 regulates slit 1 expression in the forebrain and in nonneuronal cell cultures (Yang et al., 2018).

Interestingly, our experiments suggest that despite the differential control of sema3a and slit1 by Fgfrs, downstream regulation converges along the same PI3K-Akt pathway. Similar to inhibition of Fgfr signaling, we find that multiple means of inhibiting the PI3K pathway, including pharmacological inhibition and molecular Akt gain and loss of function, reduce significantly sema3a and slit1 forebrain expression. We propose that Fgf and PI3K signaling regulate sema3a and slit1 expression cellautonomously, based on gene expression, and the speed (hours) of the changes in gene expression with pharmacological inhibition of Fgfrs (Atkinson-Leadbeater et al., 2010) and PI3K in vivo. While we cannot exclude the possibility that Fgfrs and $\mathrm{PI} 3 \mathrm{~K}$ work in parallel pathways, our data are supportive of the idea that the PI3K pathway works downstream of Fgfrs. First, pAkt analysis indicates that the $\mathrm{PI} 3 \mathrm{~K}$ pathway is regulated positively by Fgfr signaling in the forebrain. In agreement, we find that in the forebrain PI3K-Akt signaling acts on the Fgfr downstream target spry1. Second, other classical Fgfr signal transduction mechanisms do not appear to directly promote forebrain sema3a and slit1 expression, pointing to the PI3K pathway as the Fgfr signaling mediator. The PI3K pathway is known to work downstream of many extrinsic signaling molecules (Manning and Cantley, 2007; Hemmings and Restuccia, 2012), and so it is also likely that while the pathway works with Fgfrs to help maintain sema3a and slit1 expression, PI3K has other roles in the growth of axons in the forebrain, including regulating the expression of other key ligands in the brain neuroepithelium, and mediating signaling directly within axonal growth cones. In support, while the RGC axon phenotype observed with PI3K inhibition resembles that observed with Fgfr inhibition (Atkinson-Leadbeater et al., 2010), there are additional features, such as axon defasciculation.

The idea that multiple Fgfrs might work through the same PI3K pathway intermediates stands out from the current understanding of Fgfr downstream pathways. Although FGFR1-4 have similar structures, they can activate distinct sets of downstream factors and cell behaviors (Dailey et al., 2005; Ornitz and Itoh, 2015). For instance, transfection of Fgfr1 but not Fgfr4 leads to cell survival and growth of BaF3 murine lymphoid cells (Wang et al., 1994). Here, Fgfr1 strongly phosphorylates Erk, whereas Fgfr4 does not affect Erk and only weakly activates PLC $\gamma$. When Fgfrs are transfected into multiple mammalian cell lines, Fgfr $1 / 3$ strongly and consistently activate Erk $1 / 2$ and PLC $\gamma$, while Fgfr4 only weakly phosphorylates these effectors (Vainikka et al., 1994; Wang et al., 1994; Klint et al., 1995; Shaoul et al., 1995; Kanai et al., 1997; Raffioni et al., 1999). Instead, in cell lines the PI3K pathway may be the major pathway downstream of Fgfr4, as is the case for mouse NIH 3T3 fibroblasts (Vainikka et al., 1994). Thus, in contrast to the idea that differential Fgfr usage stems from a requirement for divergent downstream pathways, our data reveal that the distinct use of Fgfrs in the Xenopus forebrain to regulate different guidance genes converges on a single signal transduction pathway, PI3KAkt. What remains to be elucidated is whether Fgfr2-4 display distinct amplitudes and durations of PI3K-Akt activation and thus distinct potencies in promoting sema3a transcription.

A question that arises is why Fgfr1 does not regulate sema3a in forebrain and XTC cells, given that fgfr1 mRNA is present in both cell populations? Possibly, distinct Fgfrs access separate pools of a common downstream signaling factor. A plausible scenario is that scaffold proteins preserve the identity of the specific Fgfr from which an Fgf signal originates, even when Fgfrs share the same downstream signaling factors (Meister et al., 2013; Casar and Crespo, 2016). The Fgfr1-generated pool of PI3K may be only available to promote slit1 and not sema3a transcription. These data provide support for the idea that a signaling molecule such as PI3K may be partitioned within a 
cell based on the receptor system for which they act downstream. While multiple MAPK scaffold proteins have been identified (Casar and Crespo, 2016), we are aware of only one scaffold protein identified for PI3K-AKT, IQGAP1, which also scaffolds MAPK components under the control of epidermal growth factor receptor (Roy et al., 2005; Chen et al., 2010; Choi and Anderson, 2016, 2017).

Some studies have considered the intracellular regulation of guidance genes in cultured cell lines and after injury, but endogenous control of guidance gene expression in vivo in embryonic neuroepithelial cells has not been addressed. The in vitro studies implicate predominantly the MAPK pathway, though the time frames (days) examined are much longer than the 5-h time window we investigated. For instance, depletion of dual leucine zipper kinase, a MAPKKK that preferentially activates Jnk but may also activate Erk and p38 MAPKs, increases the expression of guidance receptors Epha7, Nrp1, Plxna4, and Unc5a, and downregulates the expression of the ligand Sema6b in cultured mouse Neuro-2a neuroblastoma cells (Blondeau et al., 2016). Further, in an ischemic retinopathy model, Interleukin 1 Beta activation of proteinase-activated receptor-2 works through Erk/Jnk in mouse RGCs to decrease Sema3a expression (Sitaras et al., 2015). Inhibition of the MAPK pathway (short or long term), however, has no effect on Xenopus embryonic forebrain sema3a and slit1 levels. Yet, activation of the MAPK pathway is sufficient to repress sema3a and slit1 transcription. These data argue that the MAPK pathway may only fine tune forebrain guidance cue levels rather than maintain expression. Since Fgf signaling increases sema3a and slit1 expression (Atkinson-Leadbeater et al., 2010), it seems unlikely that MAPK silences sema3a and slit1 downstream of Fgfrs but more likely downstream of other extrinsic molecule(s).

Guidance molecules have broad functions in development within and outside of the nervous system, and also in cancer (Chédotal et al., 2005; O'Donnell et al., 2009; Law and Lee, 2012; Luukko and Kettunen, 2016). This is true of the family of secreted Sema3s which similar to what we find for Xenopus RGCs (Atkinson-Leadbeater et al., 2010; Kita et al., 2013), function to repel and attract axons of distinct neuron types in various species (Fujisawa, 2004; Roth et al., 2009), as well as to promote axonal branching (Campbell et al., 2001) and to stimulate dendrite growth (Fenstermaker et al., 2004). Sema3s also mediate cardiovascular and bone development, lung branching, podocyte differentiation, and endothelial cell behavior (Feiner et al., 2001; Kagoshima and Ito, 2001; Yazdani and Terman, 2006; Reidy and Tufro, 2011) and can be either up- or downregulated in cancers (Chédotal et al., 2005). Thus, understanding how guidance cues like Sema3s are regulated is important given the diverse roles of these proteins in the developing and adult organism.

Axons do not rely on a single axon guidance cue to make turning decisions at guidance choice points. Instead, the actions of a large number of guidance ligands work cooperatively to ensure the proper behavior of axons. Our data indicate that at least for two guidance cues that work cooperatively to push RGC axons out of a mid-diencephalic intermediate target and on toward their final target, similar signaling mechanisms have evolved to control their expression. In brain neuroepithelial cells, the activation of Fgfrs and PI3K maintains Slit1 and Sema3a repellents in the guidance map within the mid-diencephalon. By having a common signaling mechanism, presumably the coordinated expression and function of such cues is facilitated. Multiple extrinsic factors likely define the precise spatiotemporal expression domains of sema3a, slit1, and other ligands important for guiding axons within the forebrain. Indeed, the fact that slit2 mRNA within the diencephalon is not regulated by either PI3K or Fgfrs points to additional extrinsic factors and signaling mechanisms that control guidance cue expression, distinct from those regulating sema3a and slit1.

\section{References}

Aksamitiene E, Kiyatkin A, Kholodenko BN (2012) Cross-talk between mitogenic Ras/MAPK and survival PI3K/Akt pathways: a fine balance. Biochem Soc Trans 40:139-146.

Alessi DR, Cohen P (1998) Mechanism of activation and function of protein kinase B. Curr Opin Genet Dev 8:55-62.

Alessi DR, Andjelkovic M, Caudwell B, Cron P, Morrice N, Cohen P, Hemmings BA (1996) Mechanism of activation of protein kinase $B$ by insulin and IGF-1. EMBO J 15:6541-6551.

Atkinson-Leadbeater K, Bertolesi GE, Johnston JA, Hehr CL, McFarlane S (2009) FGF receptor dependent regulation of Lhx9 expression in the developing nervous system. Dev Dyn 238:367-375.

Atkinson-Leadbeater K, Bertolesi GE, Hehr CL, Webber CA, Cechmanek PB, McFarlane S (2010) Dynamic expression of axon guidance cues required for optic tract development is controlled by fibroblast growth factor signaling. J Neurosci 30:685-693.

Atkinson-Leadbeater K, Hehr C, McFarlane S (2014) Fgfr signaling is required as the early eye field forms to promote later patterning and morphogenesis of the eye. Dev Dyn 243:663-675.

Belgacem YH, Borodinsky LN (2011) Sonic hedgehog signaling is decoded by calcium spike activity in the developing spinal cord. Proc Natl Acad Sci USA 108:482-4487.

Ben Messaoud N, Yue J, Valent D, Katzarova I, López JM (2015) Osmostress-induced apoptosis in Xenopus oocytes: role of stress protein kinases, calpains and Smac/DIABLO. PLoS One 10: e0124482.

Bertolesi GE, Su HY, Michaiel G, Dueck SM, Hehr CL, McFarlane S (2011) Two promoters with distinct activities in different tissues drive the expression of heparanase in Xenopus. Dev Dyn 240: 2657-2672

Blondeau A, Lucier JF, Matteau D, Dumont L, Rodrigue S, Jacques PE, Blouin R (2016) Dual leucine zipper kinase regulates expression of axon guidance genes in mouse neuronal cells. Neural Dev 11:13.

Boehm JS, Zhao JJ, Yao J, Kim SY, Firestein R, Dunn IF, Sjostrom SK, Garraway LA, Weremowicz S, Richardson AL, Greulich H, Stewart CJ, Mulvey LA, Shen RR, Ambrogio L, HirozaneKishikawa T, Hill DE, Vidal M, Meyerson M, Grenier JK, et al. (2007) Integrative genomic approaches identify IKBKE as a breast cancer oncogene. Cell 129:1065-1079.

Browaeys-Poly E, Blanquart C, Perdereau D, Antoine A-F, Goenaga D, Luzy J-P, Chen H, Garbay C, Issad T, Cailliau K, Burnol A-F (2010) Grb14 inhibits FGF receptor signaling through the regulation of PLC $\gamma$ recruitment and activation. FEBS Lett 584:43834388.

Brunsdon H (2015) An analysis of FGF-regulated genes during Xenopus neural development. PhD thesis, University of York.

Casar B, Crespo P (2016) ERK signals: scaffolding scaffolds? Front Cell Dev Biol 4:49.

Campbell DS, Regan AG, Lopez JS, Tannahill D, Harris WA, Holt CE (2001) Semaphorin 3a elicits stage-dependent collapse, turning, 
and branching in Xenopus retinal growth cones. J Neurosci 21: 8538-8547.

Chédotal A, Kerjan G, Moreau-Fauvarque C (2005) The brain within the tumor: new roles for axon guidance molecules in cancers. Cell Death Differ 12:1044-1056.

Chen B, Tardell C, Higgins B, Packman K, Boylan JF, Niu H (2012) BRAFV600E Negatively Regulates the AKT Pathway in Melanoma Cell Lines. PLoS ONE 7:e42598.

Chen F, Zhu HH, Zhou LF, Wu SS, Wang J, Chen Z (2010) IQGAP1 is overexpressed in hepatocellular carcinoma and promotes cell proliferation by Akt activation. Exp Mol Med 42:477-483.

Chen YY, Hehr CL, Atkinson-Leadbeater K, Hocking JC, McFarlane $S$ (2007) Targeting of retinal axons requires the metalloproteinase ADAM10. J Neurosci 27:8448-8456.

Chien CB, Rosenthal DE, Harris WA, Holt CE (1993) Navigational errors made by growth cones without filopodia in the embryonic Xenopus brain. Neuron 11:237-251.

Choi S, Anderson RA (2016) IQGAP1 is a phosphoinositide effector and kinase scaffold. Adv Biol Regul 60:29-35.

Choi S, Anderson RA (2017) And Akt-ion! IQGAP1 in control of signaling pathways. EMBO J 36:967-969.

Dailey L, Ambrosetti D, Mansukhani A, Basilico C (2005) Mechanisms underlying differential responses to FGF signaling. Cytokine Growth Factor Rev 16:233-247.

Das D, Pal S, Maitra S (2016) Releasing prophase arrest in zebrafish oocyte: synergism between maturational steroid and Igf1. Reproduction 151:59-72.

de Ramon Francàs G, Zuñiga NR, Stoeckli ET (2017) The spinal cord shows the way - How axons navigate intermediate targets. Dev Biol 1:43-52.

Dickson BJ (2002) Molecular mechanisms of axon guidance. Science 298:1959-1964.

Faber J, Nieuwkoop P (1994) Normal table of Xenopus laevis (Daudin): a systematical and chronological survey of the development from the fertilized egg till the end of metamorphosis. New York, NY: Garland Publishing.

Feiner L, Webber AL, Brown CB, Lu MM, Jia L, Feinstein P, Mombaerts P, Epstein JA, Raper JA (2001) Targeted disruption of semaphorin $3 \mathrm{C}$ leads to persistent truncus arteriosus and aortic arch interruption. Development 128:3061-3070.

Fenstermaker V, Chen Y, Ghosh A, Yuste R (2004) Regulation of dendritic length and branching by semaphorin 3A. J Neurobiol 58:403-412.

Friesel R, Dawid I (1991) cDNA cloning and developmental expression of fibroblast growth factor receptors from Xenopus laevis. Mol Cell Biol 11:2481-2488.

Fujisawa $\mathrm{H}$ (2004) Discovery of semaphorin receptors, neuropilin and plexin, and their functions in neural development. $J$ Neurobiol 59:24-33.

Fukuchi-Shimogori T, Grove EA (2001) Neocortex patterning by the secreted signaling molecule FGF8. Science 294:1071-1074.

Goetz R, Mohammadi M (2013) Exploring mechanisms of FGF signalling through the lens of structural biology. Nat Rev Mol Cell Biol 14:166-180.

Golub R, Adelman Z, Clementi J, Weiss R, Bonasera J, Servetnick M (2000) Evolutionarily conserved and divergent expression of members of the FGF receptor family among vertebrate embryos, as revealed by FGFR expression patterns in Xenopus. Dev Genes Evol 210:345-357.

Haas K, Jensen K, Sin WC, Foa L, Cline HT (2002) Targeted electroporation in Xenopus tadpoles in vivo: from single cells to the entire brain. Differentiation 70:148-154.

Hacohen N, Kramer S, Sutherland D, Hiromi Y, Krasnow MA (1998) sprouty encodes a novel antagonist of FGF signaling that patterns apical branching of the Drosophila airways. Cell 92:253-263.

Hemmings BA, Restuccia DF (2012) PI3K-PKB/Akt pathway. Cold Spring Harb Perspect Biol 4:a011189.

Holt CE (1989) A single-cell analysis of early retinal ganglion cell differentiation in Xenopus: from soma to axon tip. J Neurosci 9:3123-3145.
Jiang Z, Price CA (2012) Differential actions of fibroblast growth factors on intracellular pathways and target gene expression in bovine ovarian granulosa cells. Reproduction 144:625-632.

Jiang ZL, Ripamonte P, Buratini J, Portela VM, Price CA (2011) Fibroblast growth factor- 2 regulation of sprouty and NR4A genes in bovine ovarian granulosa cells. J Cell Physiol 226:1820-1827.

Jiminez C, Hernandez C, Pimentel B, Carrera AC (2002) The p85 regulatory subunit controls sequential activation of phosphoinositide 3-kinase by Tyr kinases and Ras. J Biol Chem 277:4155641562.

Jin Z, Chung JW, Mei W, Strack S, He C, Lau GW, Yang J (2015) Regulation of nuclear-cytoplasmic shuttling and function of Family with sequence similarity 13, member A (Fam13a), by B56containing PP2As and Akt. Mol Biol Cell 26:1160-1173.

Kagoshima M, Ito T (2001) Diverse gene expression and function of semaphorins in developing lung: positive and negative regulatory roles of semaphorins in lung branching morphogenesis. Genes Cells 6:559-571.

Kanai M, Göke M, Tsunekawa S, Podolsky DK (1997) Signal transduction pathway of human fibroblast growth factor receptor 3 . Identification of a novel $66-\mathrm{kDa}$ phosphoprotein. J Biol Chem 272:6621-6628.

Kita EM, Bertolesi GE, Hehr CL, Johnston J, McFarlane S (2013) Neuropilin-1 biases dendrite polarization in the retina. Development 140:2933-2941.

Klint P, Claesson-Welsh L (1999) Signal transduction by fibroblast growth factor receptors. Front Biosci 4:165-177.

Klint P, Kanda S, Claesson-Welsh L (1995) Shc and a novel 89-kDa component couple to the Grb2-Sos complex in fibroblast growth factor-2-stimulated cells. J Biol Chem 270:23337-23344.

Law JWS, Lee AYW (2012) The role of semaphorins and their receptors in gliomas. J Signal Transduct 2012:902854.

Lemmon MA, Schelssinger J (2010) Cell signaling by receptor tyrosine kinases. Cell 141:1117-1137.

Luukko K, Kettunen P (2016) Integration of tooth morphogenesis and innervation by local tissue interactions, signaling networks, and semaphorin 3A. Cell Adh Migr 10:618-626.

Manning BD, Cantley LC (2007) AKT/PKB signaling: navigating downstream. Cell 129:1261-1274.

Marín O, Valiente M, Ge X, Tsai L-H (2010) Guiding neuronal cell migrations. Cold Spring Harb Perspect Biol 2:a001834.

Meister M, Tomasovic A, Banning A, Tikkanen R (2013) Mitogenactivated protein (MAP) kinase scaffolding proteins: a recount. Int J Mol Sci 14:4854-4884.

Mendoza MC, Er EE, Blenis J (2011) The Ras-ERK and PI3K-mTOR pathways: cross-talk and compensation. Trends Biochem Sci 36: 320-328.

Musci TJ, Amaya E, Kirschner MW (1990) Regulation of the fibroblast growth factor receptor in early Xenopus embryos. Proc Natl Acad Sci USA 87:8365-8369.

Nakamura Y, Fukami K (2017) Regulation and physiological functions of mammalian phospholipase C. J Biochem 161:315-321.

Nakayama K, Satoh T, Igari A, Kageyama R, Nishida E (2008) FGF induces oscillations of Hes1 expression and Ras/ERK activation. Curr Biol 18:R332-R334.

O'Donnell M, Chance RK, Bashaw GJ (2009) Axon growth and guidance: receptor regulation and signal transduction. Annu Rev Neurosci 32:383-412.

Ornitz DM, Itoh N (2015) The fibroblast growth factor signaling pathway. Wiley Interdiscip Rev Dev Biol. 4:215-266.

Palmada M, Speil A, Jeyaraj S, Böhmer C, Lang F (2005) The serine/threonine kinases SGK1, 3 and PKB stimulate the amino acid transporter ASCT2. Biochem Biophys Res Commun 331:272277.

Pudney M, Varma M, Leake C (1973) Establishment of a cell line (XTC-2) from the South African clawed toad, Xenopus laevis. Experientia 29:466-467.

Raffioni S, Thomas D, Foehr ED, Thompson LM, Bradshaw RA (1999) Comparison of the intracellular signaling responses by three chi- 
meric fibroblast growth factor receptors in PC12 cells. Proc Natl Acad Sci USA 96:7178-7183.

Reidy K, Tufro A (2011) Semaphorins in kidney development and disease: modulators of ureteric bud branching, vascular morphogenesis, and podocyte-endothelial crosstalk. Pediatr Nephrol 26: 1407-1412.

Rodriguez-Viciana P, Warne PH, Dhand R, Vanhaesebroeck B, Gout I, Fry MJ, Waterfield MD, Downward J (1994) Phosphatidylinositol3-OH kinase as a direct target of Ras. Nature 370:527-532.

Roth L, Koncina E, Satkauskas S, Crémel G, Aunis D, Bagnard D (2009) The many faces of semaphorins: from development to pathology. Cell Mol Life Sci 66:649-666.

Roy M, Li Z, Sacks DB (2005) IQGAP1 is a scaffold for mitogenactivated protein kinase signaling. Mol Cell Biol 25:7940-7952.

Rubinfeld H, Seger R (2004) The ERK cascade as a prototype of MAPK signaling pathways. In: MAP kinase signaling protocols methods in molecular biology (Seger R, ed), pp 1-28. Clifton, NJ: Humana Press.

Schneider CA, Rasband WS, Eliceiri KW (2012) NIH Image to ImageJ: 25 years of image analysis. Nat Methods 9:671-675.

Seiradake E, Jones EY, Klein R (2016) Structural perspective on axon guidance. Annu Rev Cell Dev Biol 32:577-608.

Selvaraj N, Budka JA, Ferris MW, Jerde TJ, Hollenhorst PC (2014) Prostate cancer ETS rearrangements switch a cell migration gene expression program from RAS/ERK to PI3K/AKT regulation. Mol Cancer 13:61.

Shaoul E, Reich-Slotky R, Berman B, Ron D (1995) Fibroblast growth factor receptors display both common and distinct signaling pathways. Oncogene 10:1553-1561.

Sitaras S, Rivera JC, Noueihed B, Bien-Aimé M, Zaniolo K, Omri S, Hamel D, Zhu T, Hardy P, Sapieha P, Joyal JS, Chemtob S (2015) Retinal neurons curb inflammation and enhance revascularization in ischemic retinopathies via proteinase-activated receptor-2. Am J Pathol 185:581-595.

Sive H, Grainger RM, Harland RM (2000) Early development of Xenopus laevis: a laboratory manual. New York, NY: Cold Spring Harbor Laboratory Press.
Stulberg MJ, Lin A, Zhao H, Holley SA (2012) Crosstalk between Fgf and Wnt signaling in the zebrafish tailbud. Dev Biol 369:298307.

Tachibana N, Cantrup R, Dixit R, Touahri Y, Kaushik G, Zinyk D, Daftarian N, Biernaskie J, McFarlane S, Schuurmans C (2016) Pten regulates retinal amacrine cell number by modulating Akt, $\operatorname{Tgf} \beta$, and Erk signaling. J Neurosci 36:9454-9471.

Ueno H, Gunn M, Dell K, Tseng A, Williams L (1992) A truncated form of fibroblast growth factor receptor 1 inhibits signal transduction by multiple types of fibroblast growth factor receptor. J Biol Chem 267:1470-1476.

Vainikka S, Joukov V, Wennström S, Bergman M, Pelicci PG, Alitalo K (1994) Signal transduction by fibroblast growth factor receptor-4 (FGFR-4). J Biol Chem 269:18320-18326.

Vergara MN, Del Rio-Tsonis K (2009) Retinal regeneration in the Xenopus laevis tadpole: a new model system. Mol Vis 15:10001013.

Wang JK, Gao G, Goldfarb M (1994) Fibroblast growth factor receptors have different signaling and mitogenic potentials. Mol Cell Biol 14:181-188.

Webber CA, Chen YY, Hehr CL, Johnston J, McFarlane S (2005) Multiple signaling pathways regulate FGF-2-induced retinal ganglion cell neurite extension and growth cone guidance. Mol Cell Neurosci 30:37-47.

Westfall TA, Hjertos B, Slusarski DC (2003) Requirement for intracellular calcium modulation in zebrafish dorsal-ventral patterning. Dev Biol 259:380-391.

Wiedemann M, Trueb B (2000) Characterization of a novel protein (FGFRL1) from human cartilage related to FGF receptors. Genomics 69:275-279.

Yang JJ, Bertolesi GE, Hehr CL, Johnston J, McFarlane S (2018) Fibroblast growth factor receptor 1 signaling transcriptionally regulates the axon guidance cue slit1. Cell Mol Life Sci 75:36493661.

Yazdani U, Terman JR (2006) The semaphorins. Genome Biol 7:211. Zimmermann S, Moelling K (1999) Phosphorylation and regulation of Raf by Akt (protein kinase B). Science 286:1741-1744. 